\title{
Characterizing quasi-biweekly variability of the Asian monsoon anticyclone using potential vorticity and large-scale geopotential height field
}

\author{
Arata Amemiya ${ }^{1}$ and Kaoru Sato ${ }^{2}$ \\ ${ }^{1}$ RIKEN Center for Computational Science, Kobe, Japan \\ ${ }^{2}$ Department of Earth and Planetary Science, Graduate School of Science, The University of Tokyo, Tokyo, Japan
}

Correspondence: Arata Amemiya (arata.amemiya@riken.jp)

Received: 29 April 2020 - Discussion started: 19 May 2020

Revised: 25 September 2020 - Accepted: 2 October 2020 - Published: 18 November 2020

\begin{abstract}
The spatial pattern of subseasonal variability of the Asian monsoon anticyclone is analyzed using longterm reanalysis data, focusing on the large-scale longitudinal movement. The air inside the anticyclone is quantified by a thickness-weighted low-PV (potential vorticity) area on an isentropic surface. It is shown that the longitudinal movement of the air inside the Asian monsoon anticyclone has a timescale of 1 to 2 weeks, which is shorter than the monthly dominant timescale of the variability in the anticyclone intensity. The movement of the anticyclonic air is suggested to be largely controlled by passive advection. The typical time evolution of the variability pattern, explained by two leading empirical orthogonal function (EOF) components of $100 \mathrm{hPa}$ geopotential height, shows large-scale geopotential anomalies moving westward spanning from low to middle latitudes. This corresponds well with the rapid westward movement of low-PV air known as "eddy shedding" and following the eastward retreat of the anticyclonic air. The two EOF components can also explain the bimodal longitudinal distribution of geopotential maximum location.
\end{abstract}

\section{Introduction}

The Asian monsoon anticyclone (hereafter AMA; also known as the South Asian high or the Tibetan high) is characterized by a planetary-scale anticyclonic circulation, which persists in the upper troposphere and lower stratosphere (UTLS) region over the Eurasian continent throughout the northern summer. It is primarily driven by the upper level di- vergence associated with an extensive latent heat release over the southeast and south Asia induced by the monsoonal deep convection. Recently, this topic has attracted increasing attention regarding its important role in the tracer transport between the troposphere and the stratosphere. The Asian summer monsoon region is considered one of the most important pathways of tropospheric tracers entering into the stratosphere. Persistent deep convection transports the air from the boundary layer to the upper troposphere, where the air is mostly confined within the AMA (Dunkerton, 1995; Dethof et al., 1999; Gettelman et al., 2004). The anomalies of various kinds of tropospheric tracers have been observed by satellitebased instruments (Randel and Park, 2006; Park et al., 2007, 2008; Randel et al., 2010; Luo et al., 2018; Santee et al., 2017) as well as in situ measurements by water vapor and ozone sondes (Bian et al., 2012) and aircraft (Gottschaldt et al., 2018). The processes responsible for the tracer transport from the AMA to the lower stratosphere have been intensively studied in recent years using chemical transport models (Park et al., 2009; Vogel et al., 2014, 2016; Pan et al., 2016; Vogel et al., 2019) and trajectory models (Chen et al., 2012; Garny and Randel, 2016). The large-scale slow upwelling over the AMA is suggested to be the dominant transport process (Garny and Randel, 2016; Vogel et al., 2019). Shorter timescale processes associated with the subseasonal variability of the AMA, such as fast horizontal transport and turbulent mixing on an isentropic surface, also play important roles (Vogel et al., 2016; Pan et al., 2016; Gottschaldt et al., 2018; Fadnavis et al., 2018). 
An isentropic potential vorticity (PV) map is a useful illustration of daily evolution of the air around the tropopause, as PV can be approximately considered a passive tracer, which is conserved in inviscid and adiabatic motion (Hoskins et al., 1985). The AMA can be identified as an area of significantly low PV surrounded by a higher-PV area, which correspond to tropospheric and stratospheric air, respectively. The large PV gradient near the boundary of the anticyclone is considered a mixing barrier, which keeps the tropospheric chemical characteristic of the air inside the AMA (Ploeger et al., 2015). The method of quantifying the AMA intensity as an area of PV values below a specific threshold has been used to analyze its seasonal and subseasonal variability (Randel and Park, 2006; Garny and Randel, 2013).

Aside from the quantity of total area, the horizontal structure of the AMA seen in the low-PV area also shows a significant variability, with frequent movement, deformation, and occasional splitting. The deformation and splitting of the AMA usually occur towards the west of the AMA center and is called "eddy shedding" (Popovic and Plumb, 2001). The possibility of spontaneous generation of such a variability can be reproduced by a simple twodimensional dynamical model imposing a localized steady mass source (Hsu and Plumb, 2000). Further, Amemiya and Sato (2018) showed the characteristic longitudinally trapped spatial structure of the variability can also be explained by a two-dimensional dynamical model, with a little modification in background latitudinal thermal structure. The deformation and splitting of the anticyclone causes the horizontal stirring and irreversible mixing between the air trapped inside the anticyclone and outside stratospheric air (Pan et al., 2016; Gottschaldt et al., 2018). In addition, the shedding of the low-PV strip can occur eastward toward the western Pacific. It also contributes to the tracer transport from the AMA to the midlatitude lower stratosphere (Vogel et al., 2016; Fadnavis et al., 2018).

Another important spatial characteristic of the variability of the AMA is the longitudinal movement of the anticyclone center. The occurrence of the longitude of geopotential maximum on $100 \mathrm{hPa}$ shows a well-known bimodal distribution in a wide range of timescales (Zhang et al., 2002). The two modes are called Tibetan and Iranian modes based on the location of the maximum and usually used to classify temporal states of the anticyclone, although a recent study showed the robustness of the representation depends on the choice of reanalysis data set (Nützel et al., 2016).

Compared to the variability in the AMA intensity, which is considered to be predominantly driven by convective activity variability (Garny and Randel, 2013; Nützel et al., 2016), the mechanism of the variability involving the movement and deformation of the anticyclone has not been fully explained so far, although a few possible mechanisms have been suggested.

One mechanism is that the variability can be generated spontaneously by dynamical instability of two-dimensional flow. The strong horizontal shear of zonal wind on the northern and southern flank made the anticyclone dynamically unstable, which was originally suggested by Krishnamurti et al. (1973). The idea has been also supported by studies using nonlinear models, such as a beta-plane shallow water model with a localized steady forcing (Hsu and Plumb, 2000) and a mechanistic model in which a strong anticyclone is forced by a prescribed zonal jet over an idealized mountain (Liu et al., 2007). These studies have shown the possibility of the occurrence of westward eddy shedding without temporally varying external forcing.

Another possible mechanism is that the variability in the AMA structure including eddy shedding is forced by other localized pattern of subseasonal variability. It is suggested that the event-like westward movement of the anticyclone anomaly is often preceded by the burst of deep convection in southeast Asia (Annamalai and Slingo, 2001; Ding and Wang, 2007; Garny and Randel, 2013; Nützel et al., 2016). Ortega et al. (2017) has shown that the potential vorticity variability over the southern part of the AMA is often related to the convection variability migrating westward from the western Pacific in a quasi-biweekly (roughly every 2 weeks) timescale. Also, there is a well-known teleconnection pattern through the eastward propagation of a quasi-stationary Rossby wave train along the subtropical jet (Terao, 1998; Ding and Wang, 2005, 2007; Kosaka et al., 2009; Branstator and Teng, 2017). The variability in PV structure of the AMA could be influenced by that pattern triggered in the upstream regions. However, the relative importance of these relations and intrinsic dynamics remains largely unclear.

One of the necessary step towards the understanding of the structural variability of the AMA is to objectively describe the dominant spatial pattern and its time evolution. Although many efforts have been made to extract the dominant variability patterns in the Asian summer monsoon region, most analyses have focused on convection or the subtropical jet and not on the anticyclone in the UTLS as a main actor. The spatial characteristic of the variability in PV has been comprehensively described as westward eddy shedding. The concept of eddy shedding is a useful description of the significant event of the AMA variability, but it corresponds to only a part of time evolution, which actually takes place throughout the summer. It has not been clear yet if the structural variability of the AMA follows any particular pattern of time evolution or not.

Thus, the purpose of this study is to give a unified view of the dominant pattern of the variability of the AMA, incorporating the existing descriptions, namely, the event-like westward shedding of anticyclonic vortex with low-PV air and the longitudinal movement of the maximum geopotential height location, each of which has been separately discussed in different contexts so far. Based on that, this study attempts to give an implication for the responsible mechanism, which drives the variability. 
The remainder of this paper is organized as follows. In Sect. 2, the data and analysis methods used in this study are described. In Sect. 3, the subseasonal variability of the AMA is analyzed by the method using the low-PV area, focusing on the longitudinal structure changes. In Sect. 4, the time evolution of the dominant variability pattern of the AMA is examined using an empirical orthogonal function (EOF) decomposition of geopotential field and the relation to the pattern seen in the distribution of the low-PV area is discussed. Section 5 provides the summary of this paper and discussions regarding the mechanism of the variability and the relationship with other patterns in similar timescale found in previous studies.

\section{Data and methods}

\subsection{Reanalysis and observational data}

Dynamical variables from the ERA-Interim reanalysis data (Dee et al., 2011) at pressure levels with $1.5^{\circ} \times 1.5^{\circ}$ horizontal resolution are used for the analysis. Variables in the isentropic coordinates are obtained at every $5 \mathrm{~K}$ by a vertical interpolation of the original data. The analyzed time period is June-August of 1979 to 2016. Daily outgoing longwave radiation (OLR) data from the National Oceanic and Atmospheric Administration (NOAA) (Liebmann and Smith, 1996) for years from 1979 to 2016 are used as a proxy of convective activity.

\subsection{PV-based metrics of the anticyclone}

The intensity and longitudinal distribution of the AMA are quantified using a method based on isentropic PV in this study.

The method of identifying a vortex as an area enclosed by contours of a specific reference PV value has been originally developed for studies on the stratospheric polar vortex. It has been mainly used for two purposes. First, it provides the metric of the vortex intensity, which is directly related to irreversible time evolution, assuming that PV is approximately conserved (Butchart and Remsberg, 1986). Second, the edge of the polar vortex as a meridional transport boundary can be objectively detected as the maximum position of PV gradient (Nakamura, 1996; Nash et al., 1996). The calculation of the gradient is performed with respect to equivalent latitude (Norton, 1994). The advantage of these PV-based methods is that they can quantify the vortex intensity change due to diabatic and turbulent processes, regardless of the reversible perturbation caused by Rossby waves.

Similar methods have been applied to the analysis of the AMA, as it is a planetary-scale coherent vortex as large as the polar vortex. The total area enclosed by a specific PV contour is used to analyze the variability in the AMA intensity (Randel and Park, 2006; Garny and Randel, 2013). Also, Ploeger et al. (2015) attempted to objectively determine the location of transport barrier using PV and successfully showed that the barrier can be described using a temporal PV value in midsummer. The estimated location of barrier accords well with the position of discontinuity in the mixing ratio of atmospheric minor species observed by satellite instruments.

However, the applicability of the methods developed for the polar vortex to the AMA is not straightforward. The AMA is not a circumpolar but a zonally elongated elliptic vortex centered at low latitudes. Thus the theories underlying two-dimensional mixing barrier such as the effective diffusivity (Nakamura, 1996) are basically inapplicable. Moreover, the isentropic surfaces in the UTLS often have steep slopes and therefore show significantly different thickness between the troposphere and the stratosphere (Pan et al., 2012). This may cause the change in the area enclosed by a PV contour, even without external forcing processes such as deep convection.

The use of the total area inside a reference PV contour is an effective way to quantify the intensity of the anticyclone, as it measures the intensity of the vortex regardless of its location and structure. On the other hand, the variability in the location and structure of the AMA, which also occurs in a daily timescale and not measured by the total area, is also important. Thus, in this study, not only the total area of the low-PV air but also its longitudinal distribution is examined.

The total area as a function of time is described as $A_{\text {tot }}(t)$ in the following. The time evolution of $A_{\text {tot }}(t)$ is derived from the PV tendency equation (Butchart and Remsberg, 1986; Garny and Randel, 2013) as follows:

$$
\begin{aligned}
\frac{\mathrm{d}}{\mathrm{d} t} A_{\text {tot }}(t)_{q \leq q_{0}} & =\oint_{q=q_{0}}\left(-q \frac{\partial \dot{\theta}}{\partial \theta}+\dot{\theta} \frac{\partial q}{\partial \theta}\right) \frac{\mathrm{d} S}{\left|\nabla_{\theta} q\right|} \\
& +\int_{q \leq q_{0}} \nabla \cdot \hat{\boldsymbol{u}} \mathrm{d} A \\
& +(\text { subgrid scale mixing term }),
\end{aligned}
$$

where $\dot{\theta}$ is the potential temperature tendency by adiabatic processes, $\boldsymbol{u}$ is the horizontal wind vector, and $\mathrm{d} A$ and $\mathrm{d} S$ are respectively an area element and a line element of contours surrounding $A(t)$. Variable $\boldsymbol{u}$ is decomposed into resolved ( ) and unresolved (') components as $\boldsymbol{u}=\hat{\boldsymbol{u}}+\boldsymbol{u}^{\prime}$. The terms on the right-hand side are called the generation term, divergence term, and mixing term, respectively (Garny and Randel, 2013). Although the direct quantification of each term from gridded reanalysis data is difficult, it was shown by Garny and Randel (2013) using a free-running general circulation model that the first and second terms are well correlated, and hence the second term alone can be used as a proxy of convective activity.

To quantify the longitudinal movement of the low-PV air, a function of longitude and time $L(\lambda, t)$, which has a unit of length, is defined so that its longitudinal integration gives a low-PV area. 


$$
A(t)_{q \leq q_{0}}=\int_{\lambda_{\mathrm{w}}}^{\lambda_{\mathrm{e}}} L(\lambda, t) \mathrm{d} \lambda
$$

where $\lambda_{\mathrm{w}}$ and $\lambda_{\mathrm{e}}$ are arbitrary longitudes. The partial area of low-PV air of the AMA on the west of a specific longitude $\lambda_{0}$ is obtained by the integration from the western boundary of the calculation domain to $\lambda_{0}$ denoted as $A_{\text {west }}(t)$. The integration over the entire domain produces $A_{\text {tot }}(t)$.

Another treatment that is newly introduced in this study is the weighting of the low-PV area by equivalent thickness in isentropic coordinates $\sigma=-g^{-1} \partial p / \partial \theta$. The large variation of $\sigma$ in time and latitude significantly modifies the budget calculation both in seasonal mean and in subseasonal variability, as was indicated by Pan et al. (2012). In this study, instead of using $L$ and $A$, thickness-weighted quantities denoted by $\hat{L}$ and $\hat{A}$ are used. The weighted low-PV area $\hat{A}$ is defined as follows:

$$
\hat{A}(t)_{q \leq q_{0}} \equiv \int_{q \leq q_{0}} \sigma \mathrm{d} A=\iint \sigma r^{2} \cos \phi \mathrm{d} \lambda \mathrm{d} \phi,
$$

where $r$ is the radius of the Earth. $\hat{A}$ has an unit of mass divided by a temperature unit. Thus this quantity can be interpreted as a total mass of the air inside the AMA on an isentropic layer. $\hat{L}$ is related to $\hat{A}$ in a similar way as follows:

$\hat{A}(t)_{q \leq q_{0}}=\int_{\lambda_{\mathrm{w}}}^{\lambda_{\mathrm{e}}} \hat{L}(\lambda, t) \mathrm{d} \lambda$.

The equation for the tendency of mass-weighted total area $\hat{A}_{\text {tot }}(t)$ takes the following simpler form. See Appendix B for the derivation.

$$
\begin{aligned}
\frac{\mathrm{d}}{\mathrm{d} t} \hat{A}_{\mathrm{tot}}(t) & =\oint_{q=q_{0}}\left(-q \frac{\partial \dot{\theta}}{\partial \theta}+\dot{\theta} \frac{\partial q}{\partial \theta}\right) \frac{\sigma \mathrm{d} S}{\left|\nabla_{\theta} q\right|} \\
& -\int_{q \leq q_{0}} \frac{\partial}{\partial \theta}(\sigma \dot{\theta}) \mathrm{d} A+\text { (unresolved term). }
\end{aligned}
$$

Further, the budget of the mass-weighted partial area to the west of specific longitude $\lambda_{0}$ changes due to the massweighted zonal flux of low-PV air $\hat{F}\left(\lambda_{0}\right)$, in addition to the nonconservative terms:

$$
\begin{aligned}
& \frac{\mathrm{d}}{\mathrm{d} t} \hat{A}_{\text {west }}(t)=-\hat{F}\left(\lambda_{0}\right)+\oint_{q=q_{0}, \lambda \leq \lambda_{0}}\left(-q \frac{\partial \dot{\theta}}{\partial \theta}+\dot{\theta} \frac{\partial q}{\partial \theta}\right) \sigma \mathrm{d} S \\
& -\int_{q \leq q_{0}, \lambda \leq \lambda_{0}} \frac{\partial}{\partial \theta}(\sigma \dot{\theta})+(\text { unresolved term }), \\
& \hat{F}(\lambda)=\int_{q \leq q_{0}} u \sigma r \mathrm{~d} \phi
\end{aligned}
$$

The flux $\hat{F}\left(\lambda_{0}\right)$ represents the mass flux of low-PV air in the zonal direction at the longitude $\lambda_{0}$, integrated over the latitude where the PV is below the threshold. Comparing the left-hand side and the flux term on the right-hand side, the relative contribution of the movement of anticyclonic air due to conservative processes can be quantified.

The Eqs. (5) and (6) have no horizontal divergence term, which is present on the right-hand side of Eq. (1). This implies that, by the thickness weighting, $\hat{A}$ changes only when there is nonconservative forcing such as diabatic heating. Therefore, the $\hat{A}$ can be interpreted as an equivalent twodimensional representation of the air parcel. In other words, this formulation separates the effect of horizontal divergence induced by the diabatic heating from that induced by compression or tilting of the air parcel by the steep slopes of isentropic surfaces. The tendency of $\hat{A}_{\text {west }}$ is mostly explained by the longitudinal flux term $\hat{F}(\lambda)$ when other nonconservative processes over the area of $\hat{A}$ are negligible. Note that this formulation, based on the thickness-weighted area of low-PV air, is different from that based on the thickness-weighted PV budget and PV flux introduced by Ortega et al. (2018). The flux term $\hat{F}(\lambda)$ represents the mass flux of low-PV air, not the PV flux. This means, given that the threshold PV value properly reflects the mixing barrier, the flux of low-PV air implies how atmospheric chemical tracers are distributed, even when a local value of PV changes in the regions where PV is below the threshold. As an illustrative example, Fig. 1a shows a $\mathrm{PV}$ field on the $370 \mathrm{~K}$ isentropic level on a specific day. The area of PV values below 2 PVU (potential vorticity unit) is hatched by red dots. Figure $1 \mathrm{~b}$ shows the distribution of horizontal winds and thickness $\sigma$ inside the AMA. Strong anticyclonic circulation is seen along the boundary of the area. Larger values of thickness are found in the northern part of the area compared to the southern part, indicating the effectiveness of thickness weighting.

The analysis is generally sensitive to the choice of reference isentropic level, PV threshold, and calculation domain. In this study, the horizontal area $10^{\circ} \mathrm{W}-160^{\circ} \mathrm{E}, 10-50^{\circ} \mathrm{N}$ is examined. The reference PV value, isentropic surface, and the analysis domain in this study are compared to the previous studies, which used the similar method (Randel and Park, 2006; Garny and Randel, 2013; Ploeger et al., 2015) in Table 1. Budget calculations are performed for three respective isentropic levels of 360,370 , and $380 \mathrm{~K}$ using the PV thresholds shown in Table 1. A detailed analysis is mainly performed for July and August on the $370 \mathrm{~K}$ level, where the $\mathrm{PV}$ gradient is the largest. The detailed reasoning for these reference values is described in Appendix A. 
Table 1. Overview of the method of this study and previous studies which define the AMA based on the area enclosed by PV contours.

\begin{tabular}{|c|c|c|c|c|}
\hline & $\theta$ & PV & Domain & Period \\
\hline Randel and Park (2006) & $360 \mathrm{~K}$ & $\begin{array}{l}0.93 \text { PVU } \\
\text { (1.5 PVU as modified PV) }\end{array}$ & $\begin{array}{l}20-40^{\circ} \mathrm{N} \\
20-140^{\circ} \mathrm{E}\end{array}$ & $\begin{array}{l}2003 \\
\text { May-September }\end{array}$ \\
\hline Garny and Randel (2013) & $360 \mathrm{~K}$ & $0.3 \mathrm{PVU}$ & $\begin{array}{l}15-45^{\circ} \mathrm{N} \\
0-180^{\circ} \mathrm{E}\end{array}$ & $\begin{array}{l}\text { 2005-2009 } \\
\text { May-September }\end{array}$ \\
\hline Ploeger et al. (2015) & $380 \mathrm{~K}$ & $\begin{array}{l}\text { varies with time } \\
2.6-4.4 \mathrm{PVU}\end{array}$ & $\begin{array}{l}10-60^{\circ} \mathrm{N} \\
10^{\circ} \mathrm{W}-160^{\circ} \mathrm{E}\end{array}$ & $\begin{array}{l}2011-2013 \\
20 \text { June-20 August }\end{array}$ \\
\hline This study & $\begin{array}{l}360 \mathrm{~K} \\
370 \mathrm{~K} \\
380 \mathrm{~K}\end{array}$ & $\begin{array}{l}0.5 \text { PVU } \\
2.0 \text { PVU } \\
3.5 \text { PVU }\end{array}$ & $\begin{array}{l}10-50^{\circ} \mathrm{N} \\
10^{\circ} \mathrm{W}-160^{\circ} \mathrm{E}\end{array}$ & $\begin{array}{l}\text { 1979-2016 } \\
\text { June-August }\end{array}$ \\
\hline
\end{tabular}

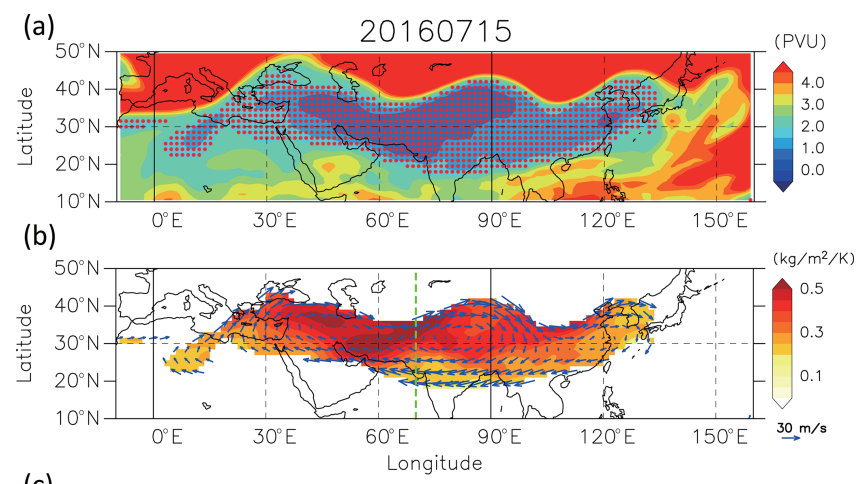

(c)

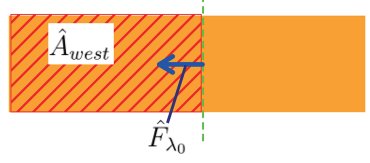

Figure 1. (a) Daily mean PV map at the $370 \mathrm{~K}$ isentropic level. The area of PV values below 2 PVU, defined as the area inside the AMA, is hatched by red dots. (b) Daily mean $\sigma$ and horizontal wind. Values are shown only for the area inside the AMA. (c) A schematic description of $\hat{A}_{\text {west }}$ and flux $\hat{F}_{\lambda_{0}}$. The reference longitude $\lambda_{0}$ is set to $70^{\circ} \mathrm{E}$ in this example.

\section{The variability of the AMA seen as the low-PV area}

\subsection{Total thickness-weighted area}

Figure 2 shows the time series of thickness-weighted low-PV area defined on 360 and $370 \mathrm{~K}$ isentropic levels, along with the time series of area-averaged OLR over $15-30^{\circ} \mathrm{N}, 60$ $120^{\circ} \mathrm{E}$ from June to August. The result for a specific year 2016 is shown as an example. Thick lines represent the lowpass-filtered 1979-2016 mean with a cutoff length of $31 \mathrm{~d}$. The mean seasonal evolution of the weighted low-PV area during summer has a peak in the middle of July. The total low-PV areas measured at 360 and $370 \mathrm{~K}$ in 2016 both fluctuate with a timescale near $30 \mathrm{~d}$ including minima in late June and late July and maxima in mid-July and mid-August.

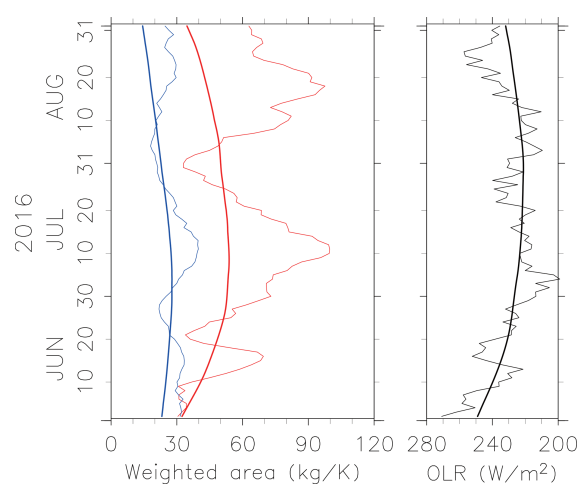

Figure 2. Daily thickness-weighted total low-PV area defined at $360 \mathrm{~K}$ (red curves) and $370 \mathrm{~K}$ (blue curves), and the OLR averaged over $15-30^{\circ} \mathrm{N}, 60-120^{\circ} \mathrm{E}$, from June to August. Thin curves represent the daily data for 2016 , and thick curves are for the $31 \mathrm{~d}$ filtered climatology.

There is also a steep maximum in the middle of June both in the low-PV area and OLR, implying an event with a shorter timescale. There is a clear correspondence between fluctuations in total low-PV area and area-averaged OLR in this year with about a few days lag, especially for $360 \mathrm{~K}$, as pointed out by previous studies (Randel and Park, 2006; Garny and Randel, 2013).

\subsection{Longitudinal movement of the air inside the AMA}

Previous studies used the total area $\hat{A}_{\text {tot }}$, without weighting, to analyze the AMA variability and found the dominant timescale around $30 \mathrm{~d}$. However, the use of total area does not take account of the zonal displacement and deformation of the AMA, which may have different timescales and patterns, such as those described as the eddy shedding. The longitudinal distribution of the low-PV area is analyzed in our study as in the following.

Figure 3 shows an average of the zonal flux of thicknessweighted area of low-PV air (Eq. 7) as well as the standard deviation as a function of the longitude on each of the 380 , 

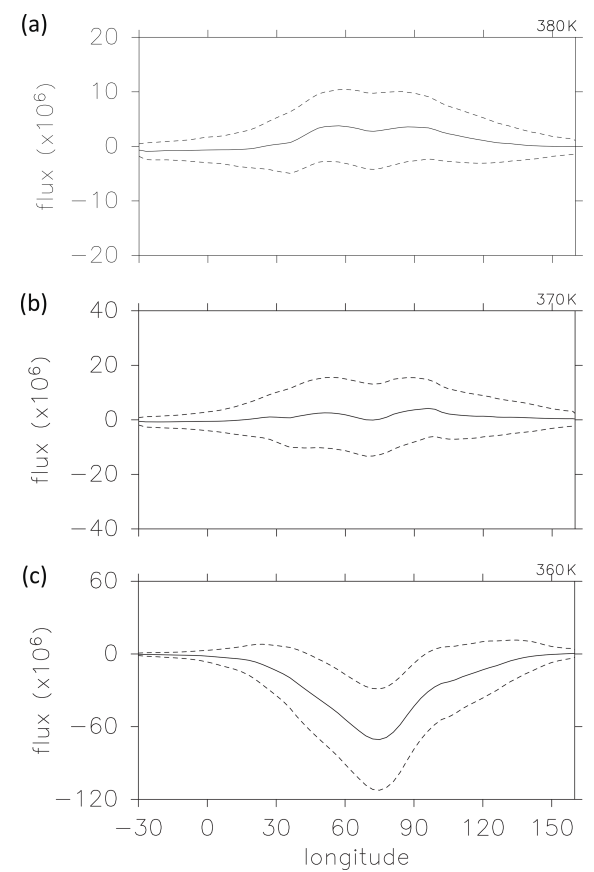

Figure 3. Climatological mean zonal flux of the thickness-weighted low-PV area as a function of longitude, calculated at the (a) $380 \mathrm{~K}$, (b) $370 \mathrm{~K}$, and (c) $360 \mathrm{~K}$ isentropic levels. The unit of the flux is kilograms per kelvin per second $\left(\mathrm{kg} \mathrm{K}^{-1} \mathrm{~s}^{-1}\right)$. Solid and broken curves correspond to mean values and ranges of standard deviations.

370, and $360 \mathrm{~K}$ level for July and August of 1979-2016. At 370 and $380 \mathrm{~K}$, the mean zonal flux is slightly eastward. The standard deviation is much larger and maximized in the longitudinal region of about $40-100^{\circ} \mathrm{E}$. This implies that the area of low-PV air oscillates zonally within these longitudes while time-mean zonal movement of air is relatively insignificant on this level. Note that the mean flux of low-PV air does not need to be zero because it can be balanced with a nonzero PV source/sink by differential heating, which is significant in seasonal mean and largely depends on the vertical levels. The important feature observed both in Fig. $3 a$ and $b$ is large fluctuations in the zonal flux of low-PV air as shown by the large standard deviations. This implies that a large part of the airmass inside the AMA can move eastward and westward. This is consistent with the results of trajectory analysis by Garny and Randel (2016), in which passive tracers released within the anticyclone tend to be trapped inside for about a month on average. At $360 \mathrm{~K}$, the mean zonal flux is largely negative between 30 and $120^{\circ} \mathrm{E}$. The positive zonal flux divergence in the eastern part is likely generated by convective forcing, while the positive flux convergence in the western part would be balanced by the sink in the low-PV area due to large vertical gradient in radiative heating/cooling around the $360 \mathrm{~K}$ level.

Figure 4 shows the longitude-time cross section of the thickness-weighted anticyclone area $\hat{A}$ calculated for each longitudinal grid interval and its zonal flux $\hat{F}(\lambda, t)$ at 360 , 370 , and $380 \mathrm{~K}$. Results for the summer months of 2016 are shown for example. Frequent zonal movement of the anticyclone air with a submonthly timescale is clearly seen. Similar longitude-time plots are found in previous studies, but most of them showed only variables averaged over a fixed range of latitude in the southern part of the AMA. Thus they may miss the longitudinal structure change after eddy shedding. The view based on the low-PV area, first introduced by Garny and Randel (2013), is useful to capture the zonal movements of the AMA regardless of its latitudinal position. Figure 4 is similar to their Fig. 6, except that the weighted values are used and the zonal flux $\hat{F}$ is added. At $360 \mathrm{~K}$, the longitudinal flux is mainly westward. At 370 and $380 \mathrm{~K}$, in contrast, the pulses of both westward and eastward flux occur alternately in July in the region of $30-120^{\circ} \mathrm{E}$. The budget of the low-PV air shows different characteristics at each of these levels. The alternate eastward and westward movements at $370 \mathrm{~K}$ imply that the variability is more like oscillatory behavior rather than dissipative westward eddy shedding as reproduced by a two-dimensional model (Hsu and Plumb, 2000).

The daily evolution of the time tendency of partial thickness-weighted area $\frac{\mathrm{d}}{\mathrm{d} t} \hat{A}_{\text {west }}$ at 360,370 , and $380 \mathrm{~K}$ on the west of $60^{\circ} \mathrm{E}$ and the contribution from the zonal flux $-\hat{F}$ through that longitude are shown in Fig. 5. At $370 \mathrm{~K}$, the two curves correspond with each other very well, except in the beginning of June when the noise in PV-based definition around the southern boundary could be large. The correlation coefficient between these two terms calculated from the time series for 1979-2016 is as high as 0.663 . This result means that the oscillation of the AMA at this level is mostly due to a simple zonal advection, and other effects such as dissipation by turbulent mixing are secondary. At $360 \mathrm{~K}$, the two curves still show the variability synchronized with each other, but with a large offset. This implies the existence of a large sink of the low-PV area at $360 \mathrm{~K}$. At $380 \mathrm{~K}$, the two curves also accord well with each other, although large disagreements are occasionally observed. This may imply sporadic nonconservative processes at this level. Note that the disagreement may be just due to the error in calculating $\hat{A}_{\text {west }}$, which is more likely to occur at $380 \mathrm{~K}$ than at lower levels (see Appendix A). While the whole picture to explain the budget of the low-PV area at these levels is more complicated than what is seen at a single level, we consider that the longitudinal oscillatory behavior with a submonthly timescale is one of the important features of the variability of AMA and the zonal flux $\hat{F}_{\lambda=60^{\circ}}$ at $370 \mathrm{~K}$ as a representative variable for it. This variable will be used as a proxy of the zonal movement of the AMA in the composite analysis in next section.

Figure 6 shows the power spectrum at $370 \mathrm{~K}$ of the zonal flux $\hat{F}_{\lambda=60^{\circ}}$ as a 38 -year mean. There is a broad peak between about 9 and $20 \mathrm{~d}$, and no peak is found around $30 \mathrm{~d}$, which corresponds to the dominant period of the variability of the AMA intensity shown in Garny and Randel (2013). This supports the idea that the variability pattern with the 

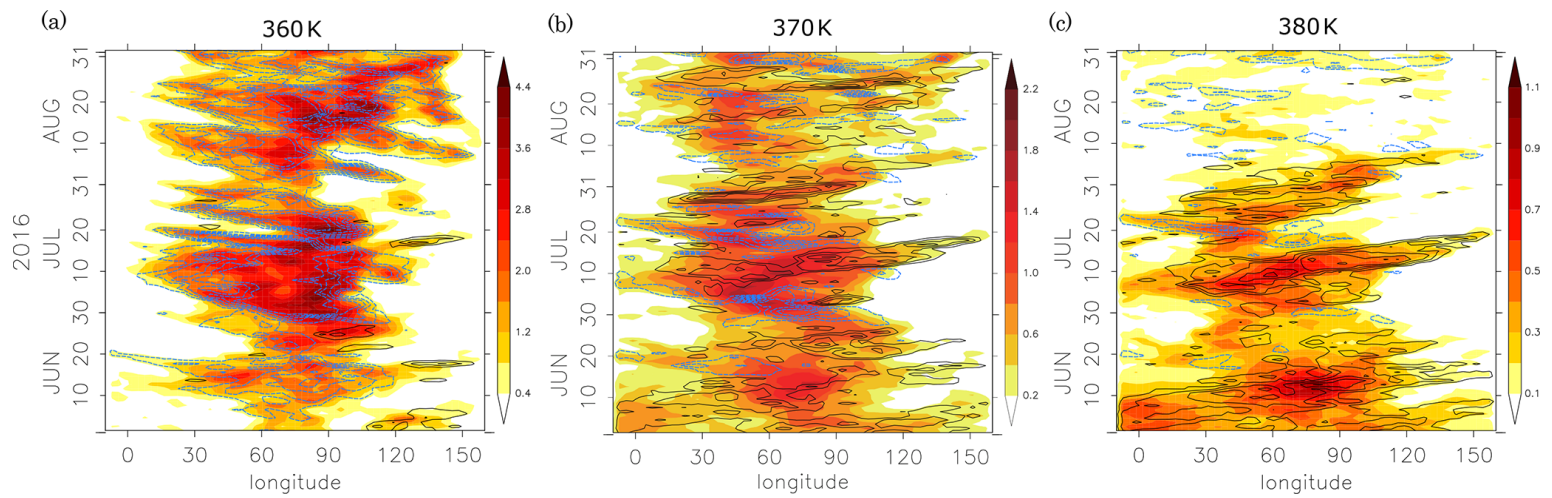

Figure 4. Time-longitude cross section of the air inside the AMA, defined based on the isentropic PV map at the (a) $360 \mathrm{~K}$, (b) $370 \mathrm{~K}$, and (c) $380 \mathrm{~K}$ levels. Color shadings show the thickness-weighted low-PV area $\left(10^{12} \mathrm{~kg} \mathrm{~K}^{-1}\right)$ calculated for each longitude grid (1.5 $5^{\circ}$ resolution). Black solid and blue dashed contours respectively show the positive and negative zonal flux of thickness-weighted low-PV area. The contour interval is $4.0,1.5$, and $1.0 \times 10^{7} \mathrm{~kg} \mathrm{~K}^{-1} \mathrm{~s}^{-1}$ in panels (a), (b), and (c), respectively.
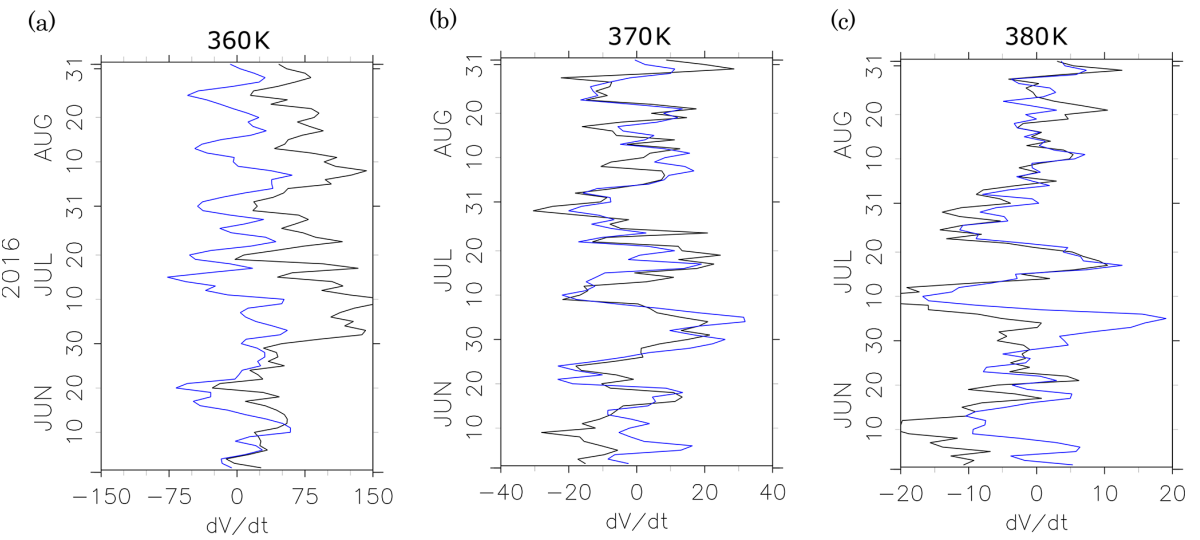

Figure 5. The tendency of the partial thickness-weighted anticyclone area $\frac{\mathrm{d}}{\mathrm{d} t} \hat{A}_{\text {west }}$ (blue: the left-hand side of Eq. 6) and westward zonal flux entering the area $-\hat{F}$ at $60^{\circ} \mathrm{E}$ (black: the first term on the right-hand side) calculated at the (a) $360 \mathrm{~K}$, (b) $370 \mathrm{~K}$, and (c) $380 \mathrm{~K}$ levels. The unit of the horizontal scale is $10^{6} \mathrm{~kg} \mathrm{~K}^{-1} \mathrm{~s}^{-1}$.

zonal movement of the AMA is effectively separated by extracting short-period components of the variability, including the quasi-biweekly timescale mentioned in previous studies. For this reason, the quasi-biweekly timescale is focused on in the following analyses.

\section{The life cycle of quasi-biweekly oscillation of the anticyclone}

\subsection{EOF decomposition}

The life cycle of the dominant large-scale pattern of the subseasonal variability of the AMA is examined using the empirical orthogonal function (EOF) decomposition. The EOF analysis is applied to the daily mean geopotential anomaly in the domain covering the AMA using ERA-Interim reanalysis data. Before calculating EOFs, anomalies are filtered with a band-pass filter within time periods of 5-20 d, normalized

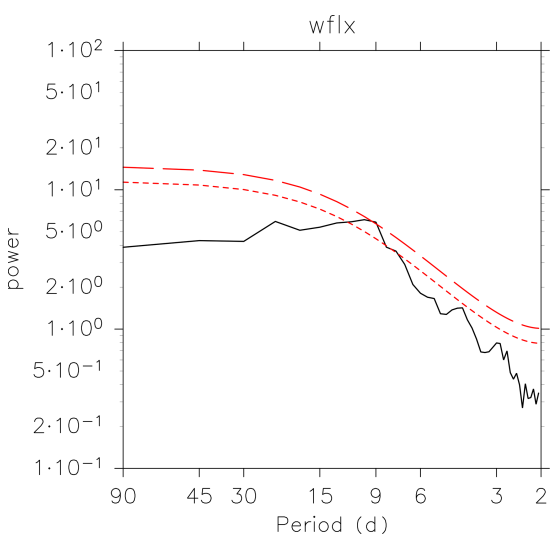

Figure 6. Power spectrum of the longitudinal flux of thicknessweighted low-PV area at $60^{\circ} \mathrm{E}$ at the $370 \mathrm{~K}$ level, calculated for June-August and averaged over 1979-2016. Broken red curves represent $95 \%$ and $99 \%$ confidence levels. 
(a)

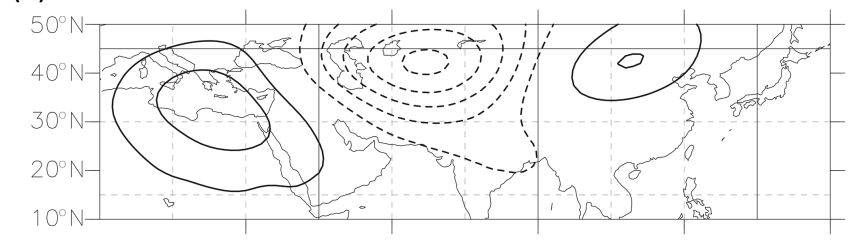

(b)

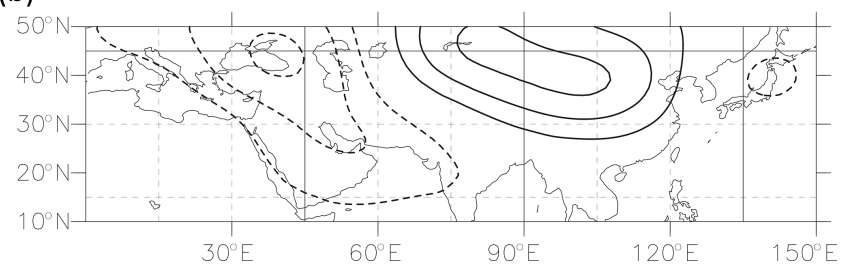

Figure 7. Spatial structure of (a) EOF1 and (b) EOF2 modes in geopotential height. The contour interval is $6 \mathrm{~m}$. Zero contours are suppressed.

at each grid point, divided by their standard deviations and weighted by the square root of grid areas. In other words, decomposition is performed for the correlation matrix weighted by each grid area instead of the covariance matrix. The normalization is effective to reduce the effect of the latitudinal dependence of geopotential height perturbation amplitude. Otherwise the perturbation patterns would be concentrated at midlatitudes, and coherent low-latitude features may be missed. The analysis is made for the regions of $0-150^{\circ} \mathrm{E}, 10$ $50^{\circ} \mathrm{N}$ at $100 \mathrm{hPa}$ and July and August of 1979-2016. EOFs for a longer period such as June to September, for slightly different levels such as 150 and $200 \mathrm{hPa}$, or for a slightly different horizontal domain do not differ much. It was confirmed that extended EOF and complex EOF analysis provides essentially similar spatial patterns (not shown). Thus, only the results obtained by a standard EOF analysis are shown.

The first two EOF components are dominant and sufficiently separated from others by North's rule of thumb (North et al., 1982). The partial variance explained by those two components are about $15 \%$ and $13 \%$, respectively. The spatial structures of geopotential height of the two components are shown in Fig. 7. Both EOF1 and EOF2 have largescale longitudinal wavy patterns approximately from 0 to $120^{\circ} \mathrm{E}$ over low and midlatitudes. The combination of these two components explains about $30 \%$ of zonally averaged total perturbation variance. Note that this latitudinal structure having large amplitudes both in low and midlatitudes is different from well-known patterns extracted by EOFs for meridional wind perturbations (Kosaka et al., 2009) or the composite analysis for the extreme events of positive geopotential height anomaly averaged over $35-45^{\circ} \mathrm{N}, 55-75^{\circ} \mathrm{E}$ (Ding and Wang, 2007). The pattern extracted in this study shown in Fig. 7 has larger scales than those previous studies. This is likely due to the choice of geopotential height anomaly for the EOF analysis, which tends to favor largerscale structure than other variables such as meridional wind.

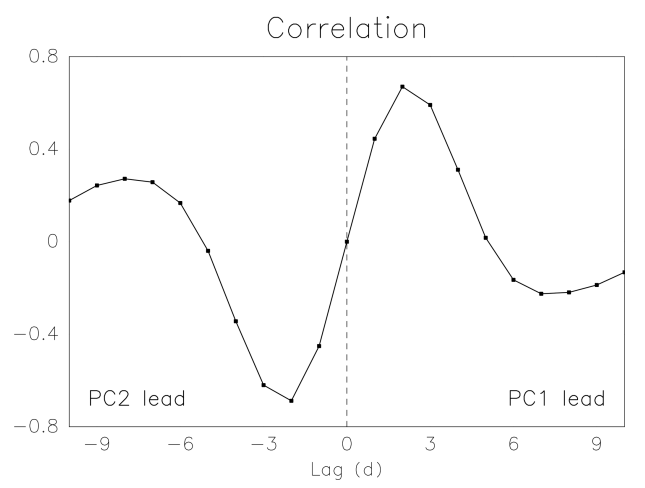

Figure 8. Lag correlation in the unit of day between the principle component corresponding to the first and second EOF modes.

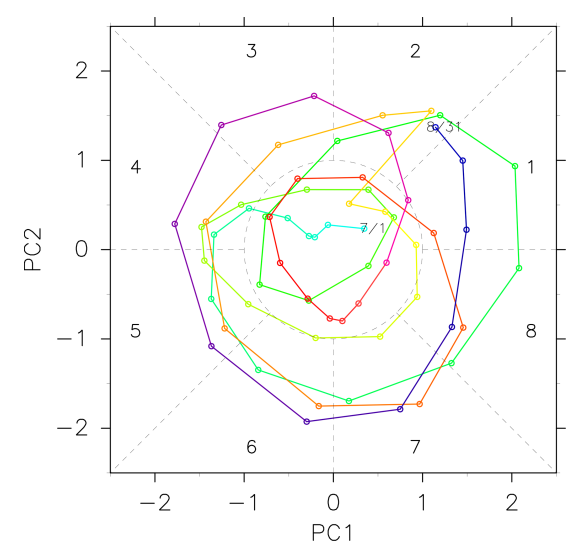

Figure 9. The definition of eight phases based on two normalized leading principal components. The daily phase progress in the year 2016 is shown as an example.

The time series of principle components (PCs) 1 and 2, corresponding to two leading EOFs, have significant lag correlation with each other, as shown in Fig. 8. PC1 is lagged behind PC2 by about $3 \mathrm{~d}$. EOF2 has the structure which is out of phase by about a quarter cycle with EOF1, indicating the dominance of westward propagation of wavy patterns.

Eight phases are defined based on normalized PC1 and 2 time series, as illustrated in Fig. 9. The daily states are labeled as each of these phases when geopotential perturbation amplitude projected onto that two-dimensional phase space exceeds unity. The summary of phase progress statistics is shown in Fig. 10. For more than $50 \%$ of cases, the phase progresses to the next within $1 \mathrm{~d}$. And for more than $60 \%$ of cases it does within $2 \mathrm{~d}$. It is also shown that the reverse progress rarely occurs. This implies the transition from a phase to the next one mostly takes place within $2 \mathrm{~d}$, which is consistent with lead time in Fig. 8 and corresponds to the quasi-biweekly dominant timescale found in Sect. 3. Based on these facts, the characteristics of the time evolution of the AMA variability pattern are examined by composite mean maps for each phase. 


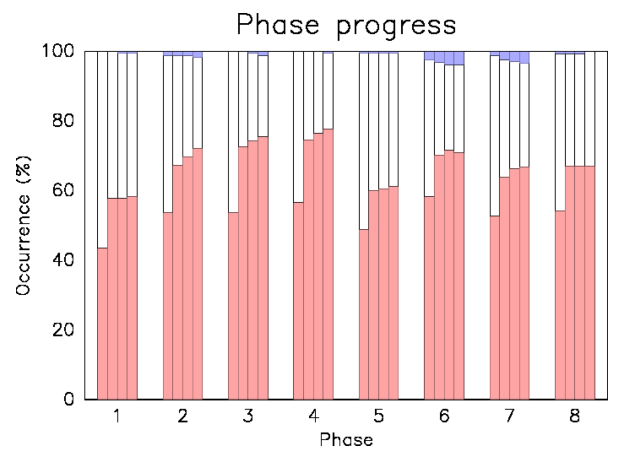

Figure 10. Percentage of forward (red) and backward (blue) phase progress starting from each phase. Four bars from left to right in each column correspond to time lags of 1 to $4 \mathrm{~d}$.

\subsection{Composite life cycle of the AMA variability pattern}

First, the life cycle of the extracted disturbance is examined in terms of the intensity, location, and structure of the AMA based on the low-PV area with a PV threshold of 2 PVU on the $370 \mathrm{~K}$ isentropic level (see Sect. 2.4). Figure 11 shows maps of the frequency of existence of the low-PV air for each phase. The areas with a percentage greater than $60 \%$ are color-shaded. As the phase progresses from phase 6 through phase 1, a large portion of the high-frequency area moves westward and reaches around $30^{\circ} \mathrm{E}$. After that, the highfrequency area drifts slightly northward and moves eastward back from phase 2 to phase 4 . This zonal oscillatory behavior is almost consistent with the large fluctuation of zonal flux of low-PV air observed in longitudes from 30 to $110^{\circ} \mathrm{E}$ in Fig. 3. The zonal movement with the phase progress is quantitatively shown as a composite mean of the thicknessweighted zonal flux of low-PV air at $370 \mathrm{~K}$ at $60^{\circ} \mathrm{E}$ (Fig. 12). The flux has a negative (i.e. westward) peak around phase 6 and positive (eastward) peak around phase 3. Figure 13 shows the mean area of the whole AMA and partial area at $370 \mathrm{~K}$ to the west of $60^{\circ} \mathrm{E}$ for each phase. The standard deviation is also shown by dashed curves. The western part fluctuates with phase, as it is largely controlled by the zonal flux in weekly timescale (Fig. 5). In contrast, the total area does not show significant dependence on the phase. As the total area is likely to be controlled by the total intensity of the thermal forcing, this implies that the variability in the thermal forcing intensity is not a key factor for this variability pattern. This supports the idea that the variability in the biweekly timescale is determined by internal dynamics.

Next, the spatial characteristics of geopotential height and the variables related to convective activities during the phase progress are examined. Composites of geopotential height (black contours) and its anomaly from climatology (color shades) on $100 \mathrm{hPa}$ along with OLR anomalies (red (positive) and green (negative) contours) are shown in Fig. 14. As already seen in Fig. 7, geopotential anomaly exhibits a large-scale wave-like pattern propagating westward along the subtropical jet about $40-50^{\circ} \mathrm{N}$. The geopotential anomalies are extended southeastward to low latitudes. The positive geopotential anomaly along $30^{\circ} \mathrm{N}$ is located at about $70^{\circ} \mathrm{E}$ at phase $5,50^{\circ} \mathrm{E}$ at phase $6,40^{\circ} \mathrm{E}$ at phase 7 , and $30^{\circ} \mathrm{E}$ at phase 8 . The westward movement of the positive geopotential anomaly follows roughly the movement of high occurrence of a low-PV area from phase 5 to phase 8 (Fig. 11).

Statistically significant OLR anomalies are found mainly over the Tibetan Plateau, southern China, and the region from the northern Bay of Bengal to northern India. The anomalies over the Tibetan Plateau are in phase with geopotential anomalies of the same sign. Over other two regions, the relation of the OLR anomalies with geopotential anomalies and with PV distribution (Fig. 11) is less clear. The observed convection variability may occur in response to the large-scale dynamical variability and/or have an influence on the dynamics. This point will be discussed in the next section.

Figure 15a and b show the geopotential anomalies averaged over $15-25$ and $35-45^{\circ} \mathrm{N}$, respectively, in the longitude-pressure cross sections. Color shading shows the geopotential anomalies from the climatology, whereas contours show the anomalies from the longitudinal mean to indicate the AMA center location. Only results for phases 1 to 4 are shown, as the features for the rest of the phases appear close to the negative counterparts. The longitude-pressure structure of the anomalies is almost barotropic in both the low and middle latitudes. The amplitude of the anomalies is maximized around $100 \mathrm{hPa}$ for the low latitudes and around $150-200 \mathrm{hPa}$ for the midlatitudes, reflecting the difference in the tropopause height. The level of the maximum roughly corresponds to the tropopause level. Thus it is considered that the pattern is essentially trapped by the tropopause, as is the AMA itself (Popovic and Plumb, 2001). For the low latitudes, significant anomalies are observed down to about $400 \mathrm{hPa}$, close to the level where the convective thermal forcing is maximized. In contrast, vertical structure is the deeper at midlatitudes.

\subsection{The relation with the bimodality in anticyclone center location}

The bimodality of the center longitude seen at $100 \mathrm{hPa}$ is one of the important characteristics of spatial variability of the AMA. The relation between the bimodality and the quasibiweekly AMA variability pattern defined in this study is examined.

Using the ERA-Interim reanalysis data from July to August of 1979-2016, the longitudinal distribution of the AMA center, which is defined as the location of daily mean geopotential height maximum, is calculated. The total distribution is shown in the bars in Fig. 16. The bimodal distribution with peaks around 60 and $90^{\circ} \mathrm{E}$ is observed. The distribution is also calculated separately for each phase, as shown in curves in different colors in Fig. 16. Numerals show the location of peak longitudes for respective phases. There is a clear phase 

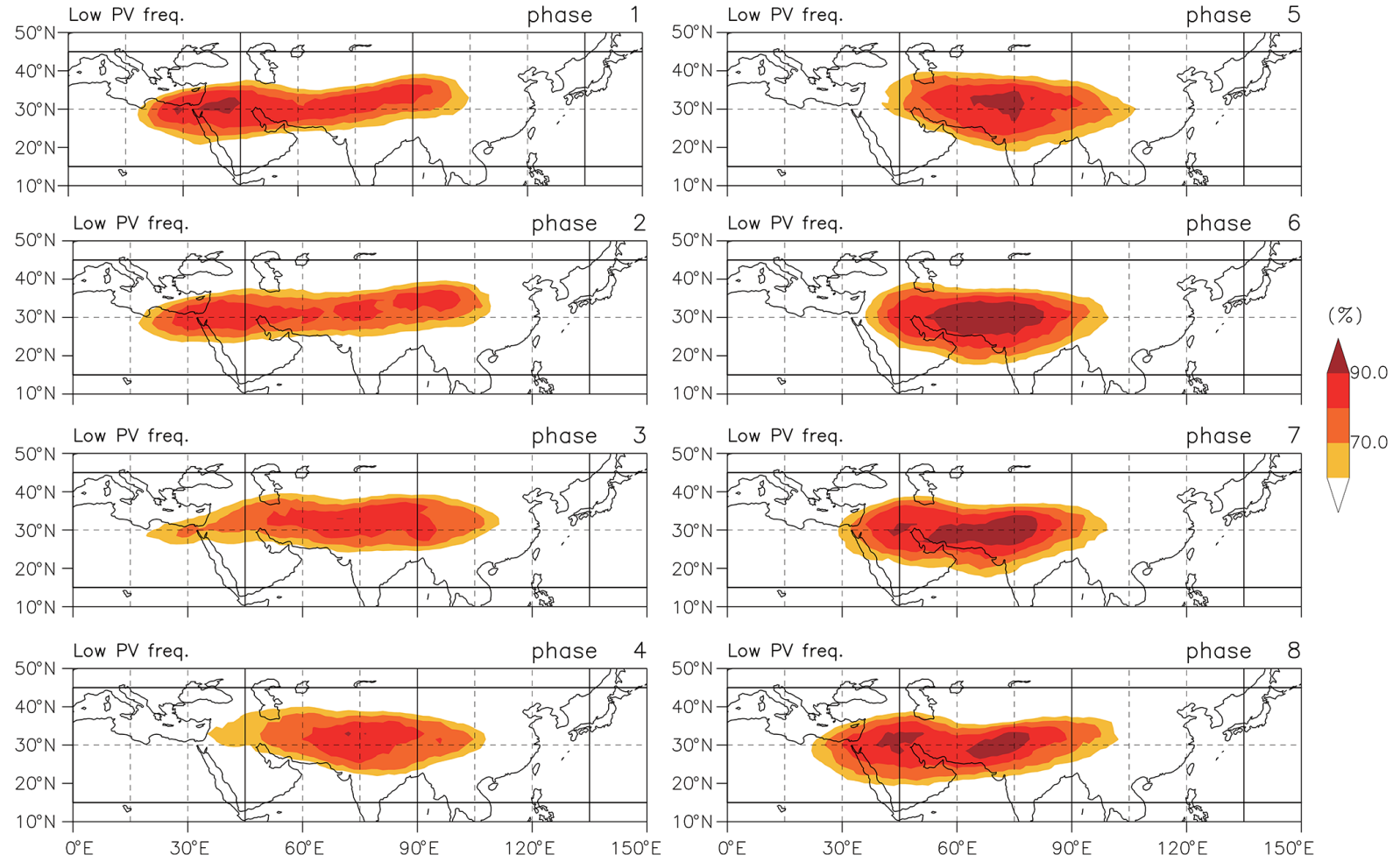

Figure 11. Percentage of PV value lower than the reference value of $2.0 \mathrm{PVU}$ at the $370 \mathrm{~K}$ isentropic level for each phase.

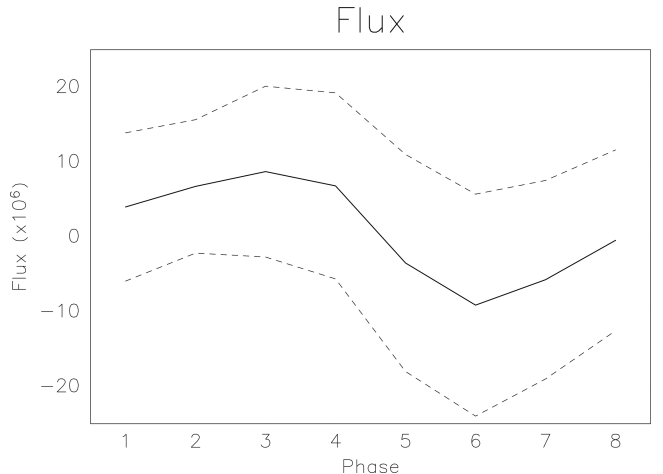

Figure 12. Mean value (solid line) and the range of standard deviation (broken lines) of the zonal flux of thickness-weighted low-PV air $\left(\mathrm{kg} \mathrm{K}^{-1} \mathrm{~s}^{-1}\right)$ at $60^{\circ} \mathrm{E}$ calculated at the $370 \mathrm{~K}$ level for each of the eight phases.

dependence of the distribution. Phases 1 to 3 favor the eastern location around $90^{\circ} \mathrm{E}$ of the AMA center, while phases 5 to 8 favor the western location around $60^{\circ} \mathrm{E}$. This phase dependence can be mostly explained by the spatial structure of EOF2 shown in Fig. 7b. The partial variance explained by EOF2 component is sufficiently large to enable large positive and negative EOF2 components to contribute to eastward and westward displacement of the AMA center, respectively.

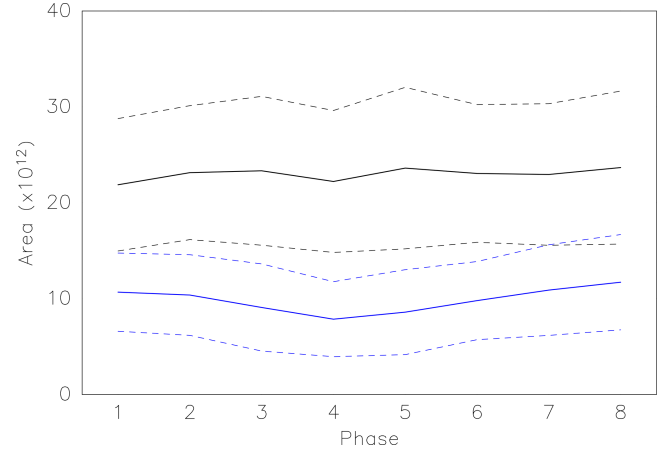

Figure 13. Mean values (solid curves) and the ranges of standard deviation (broken curves) of the area of thickness-weighted lowPV air $\left(\mathrm{kg} \mathrm{K}^{-1}\right)$ calculated at the $370 \mathrm{~K}$ level for each of the eight phases. Black and blue curves correspond to the total area and the partial area westward of $60^{\circ} \mathrm{E}$, respectively.

\section{Summary and discussion}

In this study, the subseasonal variability of the AMA, which includes longitudinal movements on a quasi-biweekly timescale, was examined. The analyses were performed from two different perspectives, that is, the movement of the air inside the AMA defined as thickness-weighted low-PV area and an EOF decomposition of normalized geopotential anomaly field on $100 \mathrm{hPa}$. 

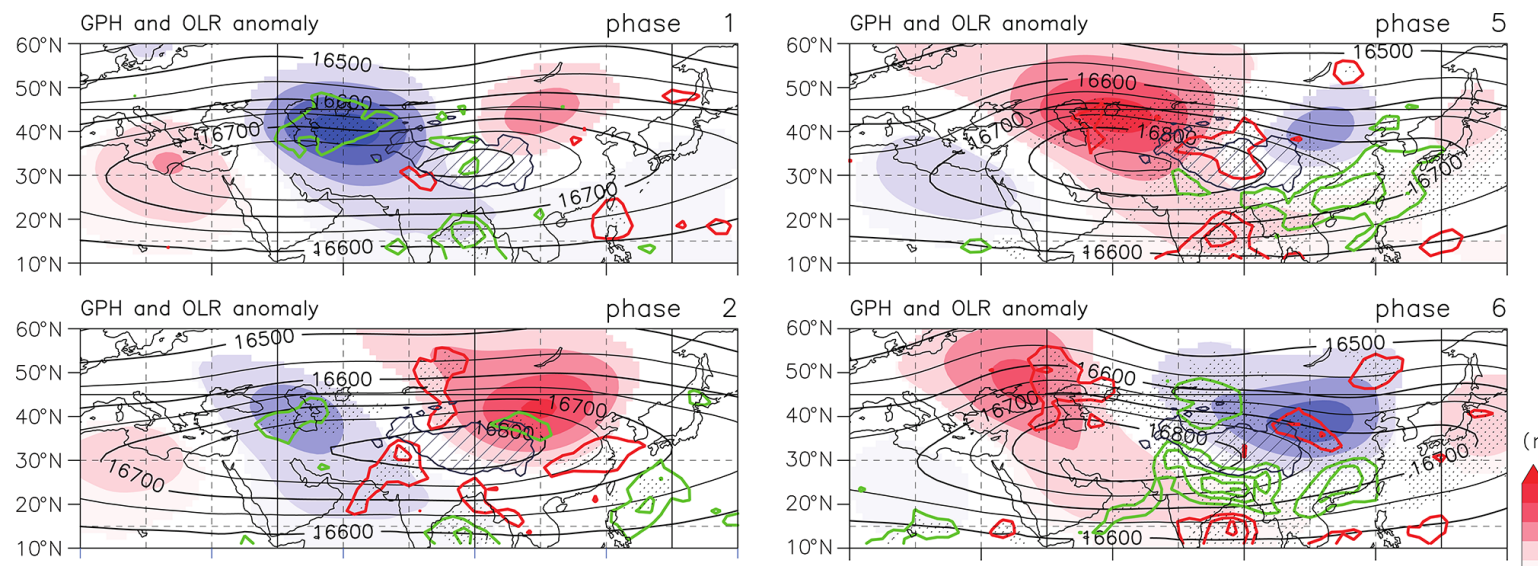

\section{0}
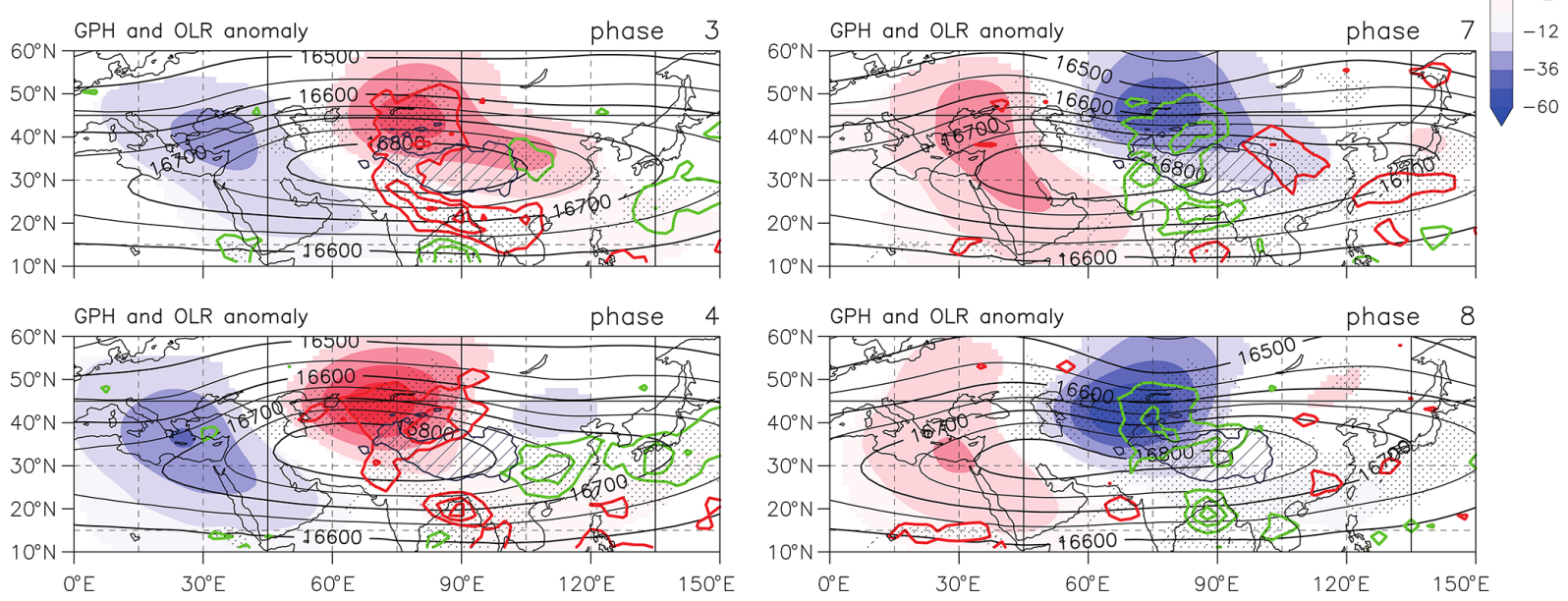

Figure 14. Composite maps of geopotential height (black contour) and its anomaly (shade) at the $100 \mathrm{hPa}$ level, and the OLR anomaly (green and red contour) for each phase. Anomalies are calculated as deviations from climatology. The contour interval for geopotential height is $50 \mathrm{~m}$. The contour interval for the OLR anomaly is $5 \mathrm{~W} \mathrm{~m}^{-2}$, with green and red contours respectively representing negative and positive anomalies. Shading of geopotential anomalies shows only areas with $95 \%$ significance by a standard $t$ test. The areas of OLR anomaly with $95 \%$ significance are hatched by dots. Areas with an elevation above $3000 \mathrm{~m}$ are hatched.

The zonal distribution of the thickness-weighted low-PV area and its zonal flux was calculated using ERA-Interim reanalysis data from 1979 to 2016. The longitudinal distribution of the low-PV area in midsummer exhibits a significant temporal fluctuation. The budget analysis revealed that the tendency of the partial thickness-weighted low-PV area on the west of the specified longitude of $60^{\circ} \mathrm{E}$ is mostly controlled by the zonal flux entering the domain on a subseasonal timescale. This suggests that the variability is mostly controlled by the large-scale dynamics, and other nonconservative processes such as turbulent dissipation and diabatic heating by radiation and/or convection have secondary roles on this timescale. Thus the variability can be characterized as event-like pulses of zonal flux of low-PV air at the longitude around $60^{\circ} \mathrm{E}$.

The large-scale variability pattern in geopotential height on a pressure level around the tropopause was reconstructed by the first two EOF components. They explained about $30 \%$ of the total variance and are significantly separated from other components. They had large-scale anomaly patterns spanning from middle to low latitudes with comparable amplitudes and a significant lead-lag correlation with each other. The reconstructed pattern by these two components showed a westward-moving large-scale geopotential anomaly. By defining the phase of this pattern based on a two-dimensional phase space by these leading modes, spatial structures of variables at each phase in the whole life cycle were examined. The distribution of the occurrence frequency of low-PV on the $370 \mathrm{~K}$ level indicated clear zonal oscillation of the air inside the AMA as the phase progresses. There was a significant relationship between the phase and zonal flux of low-PV air, as the rapid westward movement around $60^{\circ} \mathrm{E}$ at phase 6 in Fig. 11 corresponds to large westward flux of low-PV air in Fig. 12. The composite of geopotential height shows the westward-propagating large-scale anomaly pattern along the subtropical jet. The vertical structure is nearly barotropic in both middle and low latitudes. The influences down to the level of the middle troposphere were 

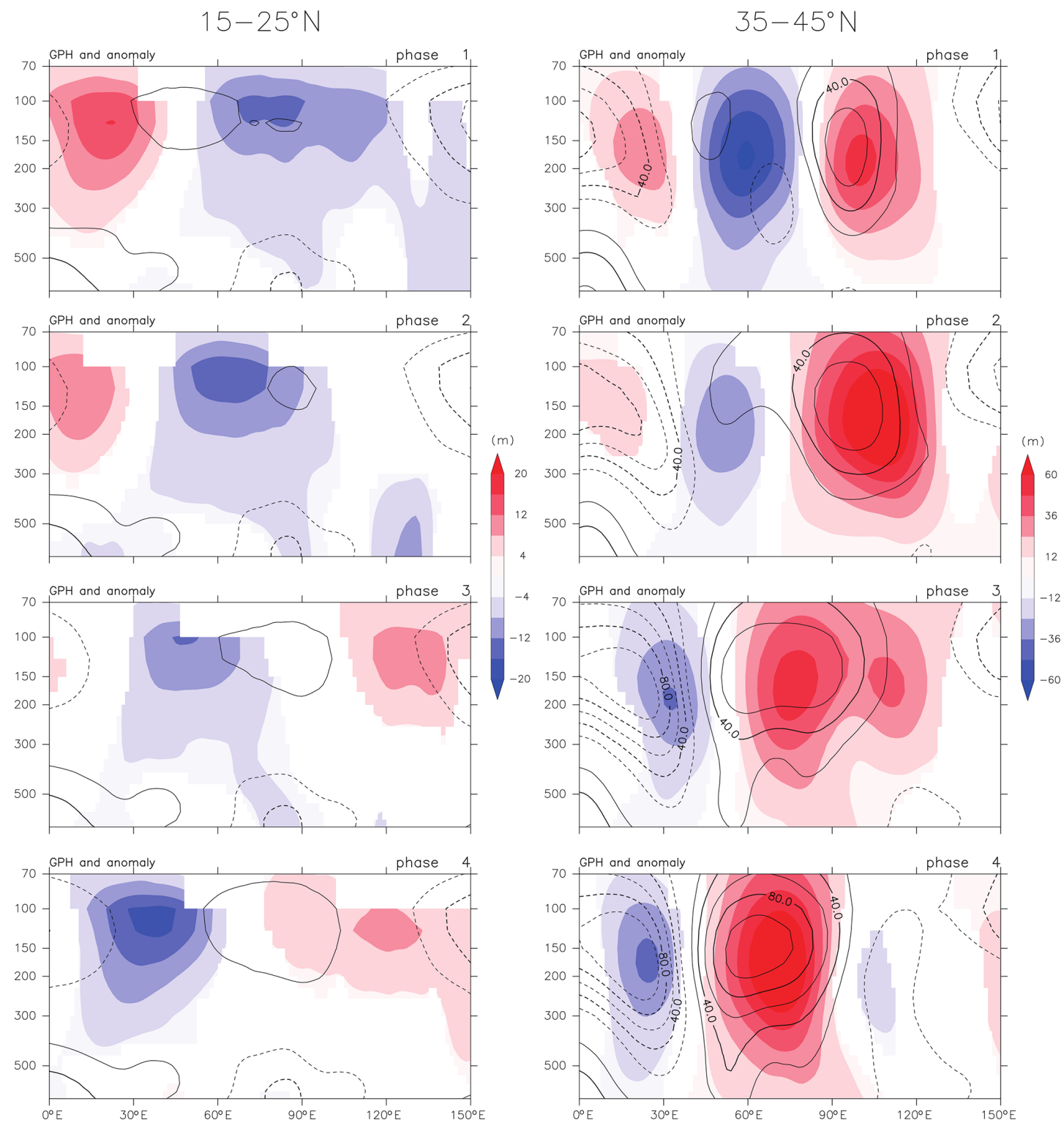

Figure 15. Composite of geopotential height in the longitude-pressure section. Contours show deviation from the zonal mean of the longitude sector, and its anomalies from climatology are shaded. The left and right columns respectively show maps averaged over $15-25$ and $35-45^{\circ} \mathrm{N}$. Only the first four of eight phases are shown. The contour interval is $20 \mathrm{~m}$. Only the areas with $95 \%$ significance, determined by standard $t$ test, of geopotential anomalies are shaded. Note that different color scales are used for the left and right columns.

observed at the midlatitude, whereas at the low latitudes disturbances are largely trapped above $300 \mathrm{hPa}$.

The variability pattern revealed in this study has a robust tendency of cyclic time evolution (Fig. 10) in which a quasibiweekly timescale is dominant. Thus an important question is what drives this variability and determines the timescale. The driver of the AMA variability has been discussed in previous studies focusing mainly on seasonal evolution and longer period variability patterns. For the variability in the anticyclone intensity with a monthly timescale, the essential role of the variability in convection in south to southeast Asia has been suggested (Garny and Randel, 2013; Nützel et al., 2016). However, it is not straightforward whether the similar relationship applies to the quasi-biweekly variability focused on in this study. The causal relationship between convective variability and the UTLS circulation variability in the quasibiweekly timescale remains an important question. There have been a few studies that suggest variability patterns coupled with convection anomalies of smaller spatial scale over the area including the Tibetan Plateau, east Asia, south Asia, and the western Pacific. For example, Fujinami and Yasunari (2004) suggested a cyclic pattern of convective anomalies 


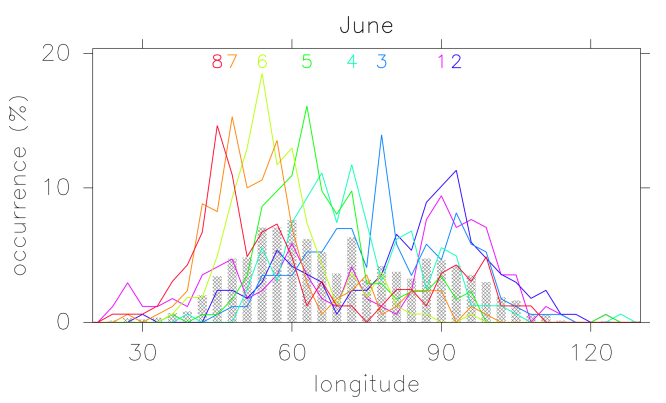

Figure 16. Longitudinal distribution of the occurrence frequency of the AMA center defined as the geopotential maximum at the $100 \mathrm{hPa}$ pressure level. Hatched bars show the total average. Lines show partial averages of the data assigned for each phase, as denoted by numbers of the corresponding colors.

propagating clockwise from Tibetan Plateau via south China and northeast India, along with a Rossby wave train over the subtropical jet. Also, a recent study by Ortega et al. (2017) has examined the coupling between quasi-biweekly variabilities in tropospheric convection and the UTLS dynamics, suggesting the possibility that the latter one leads the former. However, as they used area-averaged PV over southern India for a metric of upper-tropospheric disturbances, their results have captured the pattern significant for the southeastern part of the area of AMA, not the whole extent of AMA, which includes midlatitudes and west Asia. In this study, the statistically significant pattern of OLR anomalies was found in the composite analysis based on the EOF leading components of dynamical field perturbations (Fig. 14). This anomaly pattern of convection may be the response to the dynamical variability, or rather the driver of the variability. A recent study by Wei et al. (2019) suggested the latter possibility based on their composite analysis focusing on the longitudinal movement of the AMA center. However the spatial location of the OLR anomalies corresponding to each of the phases of the AMA variability found in this study does not necessarily support it. For example, in phase 6, there are significant negative OLR anomalies over southern China and northeastern to northern India. This feature indicates the intensified convection and the explanation of the low-PV area by uppertropospheric horizontal divergence over these areas. However, at this phase, the maximum low-PV area probability in Fig. 11 is located around $50-80^{\circ} \mathrm{E}$, to the west of the area of intensified convection. This mismatch implies that the westward movement of the low-PV area is not forced or triggered by a temporal burst of monsoonal convection over northeastern to northern India, provided that this phase progress based on the EOF analysis properly captures the characteristic time evolution of dynamical fields associated with the variability pattern. The relationship between the anomaly pattern found in this study and that of previous studies should be explored in future studies.
The subseasonal variability pattern can be driven by dynamical instability of two-dimensional anticyclonic flow, as mentioned in Sect. 1. The essentially barotropic structure of the anomalies seen in Fig. 15 supports the validity of a conceptual two-dimensional model to explain the dynamics. The analysis in this study using thickness-weighted low-PV area and its zonal flux showed the pulse of westward movement of low-PV air characterizing the variability. This behavior corresponds to spontaneous eddy shedding reproduced by the two-dimensional model in a previous study (Hsu and Plumb, 2000). However, there is an essential difference between their modeled eddy shedding and the observed variability in terms of the budget of the low-PV area. Whereas anticyclonic eddies dissipate after westward shedding in the conventional dynamical model, the low-PV area in reality does not dissipate but mostly returns eastward and forms the oscillatory pattern seen in Figs. 11 and 12. A recent study attempts to explain this behavior, using a modified two-dimensional dynamical model, which includes the effect of latitudinally varying tropopause structure (Amemiya and Sato, 2018).

The zonal oscillation of the AMA viewed as a low-PV area in this study is directly linked to the oscillation of the mixing ratio anomalies of various atmospheric minor constituents, as PV approximately acts as conserved quantity as well. Irreversible tracer transport through the AMA occurs with several dynamical or physical processes. Those are dependent on the temporal structure or position of the AMA during the subseasonal variability. For example, the largescale upwelling, which transports the air into the tropical lower stratosphere, may correspond to the temporal position of the AMA. Turbulent mixing can be enhanced in the process of eddy shedding. Also, occasional westward or eastward shedding of tropospheric air out of the AMA, which contributes to the transport to the midlatitude stratosphere (Vogel et al., 2016), may be dependent on the phase of largescale variability described in this study.

Additionally, the understanding of the dynamics of the AMA and its relation to tropospheric weather patterns is practically important. The subseasonal variability of the Asian summer monsoon is one of the most essential factors in predicting the risk of high-impact weather such as heavy rainfall and droughts in south and southeast Asia. For practical purposes, describing the dominant variability patterns and their typical time evolution provides a useful framework for a subseasonal prediction, as has been successfully applied to the Madden-Julian Oscillation. Recent studies have found several dynamical predictors for heavy rain events (Ding and Wang, 2009) and introduced real-time multivariate indices for the variability of the Asian summer monsoon (Lee et al., 2013). The pattern discussed in this study based on low-PV area and geopotential anomalies focuses on a wider area from Middle East to east Asia including the Tibetan Plateau. The relative importance of this variability pattern for local precipitation prediction and the relation to existing patterns is an interesting topic for future study. 
Appendix A: The choice of reference PV, isentropic level, and southern boundary latitude of the domain

In this study, the area of the anticyclone is defined as the area in which PV is lower than a specific reference value. The domain used for this analysis should be specified by certain ranges of longitude and latitude where the AMA is typically found. The proper choice of such longitude and latitude ranges, as well as the reference PV value and isentropic level, is not a trivial issue, because the boundary of the AMA is not always well defined by a fixed value of PV, especially on its southern flank. As seen in Fig. 1, PV values near the Equator are as low as that inside the AMA, although the equatorial UTLS air generally has a different origin and does not mix with the air inside the AMA easily, as they are usually separated by the air in low latitudes with larger PV values. Ideally, by choosing sufficiently low reference PV value and proper position of the southern boundary of the domain, the AMA can be detected as an isolated area of low-PV within the domain. However, due to the large subseasonal variability and seasonal evolution of the AMA both in PV value and structure, it is sometimes difficult to isolate the area of the AMA from the equatorial air. When the southern boundary of the domain is located too close to the Equator, the equatorial air, which is supposed to be of stratospheric origin, may be counted as the area inside the AMA. In contrast, when it is located higher than the optimal latitude, part of southern portion of the AMA will be excluded. In such a case the AMA intensity is underestimated, and the important response to the low-latitude convection might be missed. Additionally if the reference PV value is too large, it is not always possible to separate the AMA and the equatorial air by the closed PV contour. Therefore, although these sources of error can not be completely excluded, it is worthwhile to show how and to what extent they can be minimized by the optimal choices of the domain boundary and reference PV and isentropic surface values.

In the following, the largest source of error is considered to be generated from the choice of the southern boundary latitude of the domain and a reference PV value, while other boundary positions are fixed. The northern boundary is fixed at $50^{\circ} \mathrm{N}$. The eastern and western boundary are set to $160^{\circ} \mathrm{E}$ and $10^{\circ} \mathrm{W}$, respectively, following Ploeger et al. (2015). It is confirmed that the sensitivities of calculated AMA areas to these values are not significant.

The optimal southern boundary latitude and the reference PV value are explored as follows. On each of the isentropic surfaces 360,370 , and $380 \mathrm{~K}$, the frequency of occurrence of grid points with PV lower than the reference within the longitudinal range $\left(10^{\circ} \mathrm{W}\right.$ to $\left.160^{\circ} \mathrm{E}\right)$ is calculated as a function of latitude. Calculation is performed for each month from June to September, using the 6-hourly ERA-Interim reanalysis data from 1979 to 2016.

Figure A1 shows the results on each of three isentropic surfaces for each month. Generally, the occurrence of low-PV air has a peak in subtropical latitudes separated from the high occurrences near the Equator. The relative error of the AMA area calculation is implied by the significance of isolation of such a peak. Sufficiently high occurrence of PV lower than the reference value around the subtropical latitudes and infrequent occurrence on the equatorial periphery of the AMA are desirable. Such a contrast is the most clear in July and August at $370 \mathrm{~K}$, when the value around $2 \mathrm{PVU}$ is used as the reference. In June, the AMA areas are almost similar to those in July, although there are higher occurrences in low latitudes. The latitude of maximum occurrence, which roughly corresponds to the AMA center, is located southward compared to that in July, and the separation from the equatorial air is less clear. In September, the contrast in occurrence between low latitudes and midlatitudes becomes obscure for most reference PV values. Such behavior with respect to seasonal transition is consistent with the seasonal evolution of the AMA shown in monthly climatology of PV on $370 \mathrm{~K}$ isentropes in Fig. A1. The similar seasonal evolution can be observed at other isentropic levels. At $380 \mathrm{~K}$, less significant maximum occurrences in midlatitudes are found. At $360 \mathrm{~K}$, the whole pattern is shifted to lower latitudes and the minimum in low latitudes is more obscure. Considering these results, the value around 2.0 PVU on $370 \mathrm{~K}$ isentropic level is implied to be the best reference value. On 360 and $380 \mathrm{~K}$, the respective reference values should be around 0.5 PVU and 3.5 PVU to describe the anticyclonic vortex while minimizing the noise by the southern boundary.

Those values are also compared with the barrier PV values on each isentropic level, determined objectively following the method by Ploeger et al. (2015). Their criterion to detect the mixing barrier is based on the maximum of the PV gradient with respect to equivalent latitude defined based on PV (see their Sect. 4 for the detailed methodology). Using the long-term reanalysis data from 1979 to 2016, we calculated the barrier PV value for each day on each of the 360 , 370 , and $380 \mathrm{~K}$ isentropic levels.

Figure A2 summarizes the result. The median (50th percentile) and 15th and 85th percentiles of barrier PV values, along with the ratio of barrier detection in number (\%), are shown for each 10 or $11 \mathrm{~d}$ month part of the boreal summer season. We found that $370 \mathrm{~K}$ is the most favorable isentropic level to detect the barrier objectively, with percentages above $60 \%$ throughout July and August, although Ploeger et al. (2015) have shown that $380 \mathrm{~K}$ provides the most significant barrier feature. This discrepancy can be explained considering that the detectability can vary in an interannual and long-period intraseasonal timescale, reflecting large dynamical variability. On $360 \mathrm{~K}$, the percentage of barrier detection has lower values compared to 370 and $380 \mathrm{~K}$ but still larger than $40 \%$ in July. In July and August, the range of barrier $\mathrm{PV}$ values is almost unchanged with respect to time, while in June lower values are more dominant. Median PV values on each of the 360,370, and $380 \mathrm{~K}$ levels are near $0.5,2.0$, and 3.5 PVU, respectively. These values are close to optimal val- 

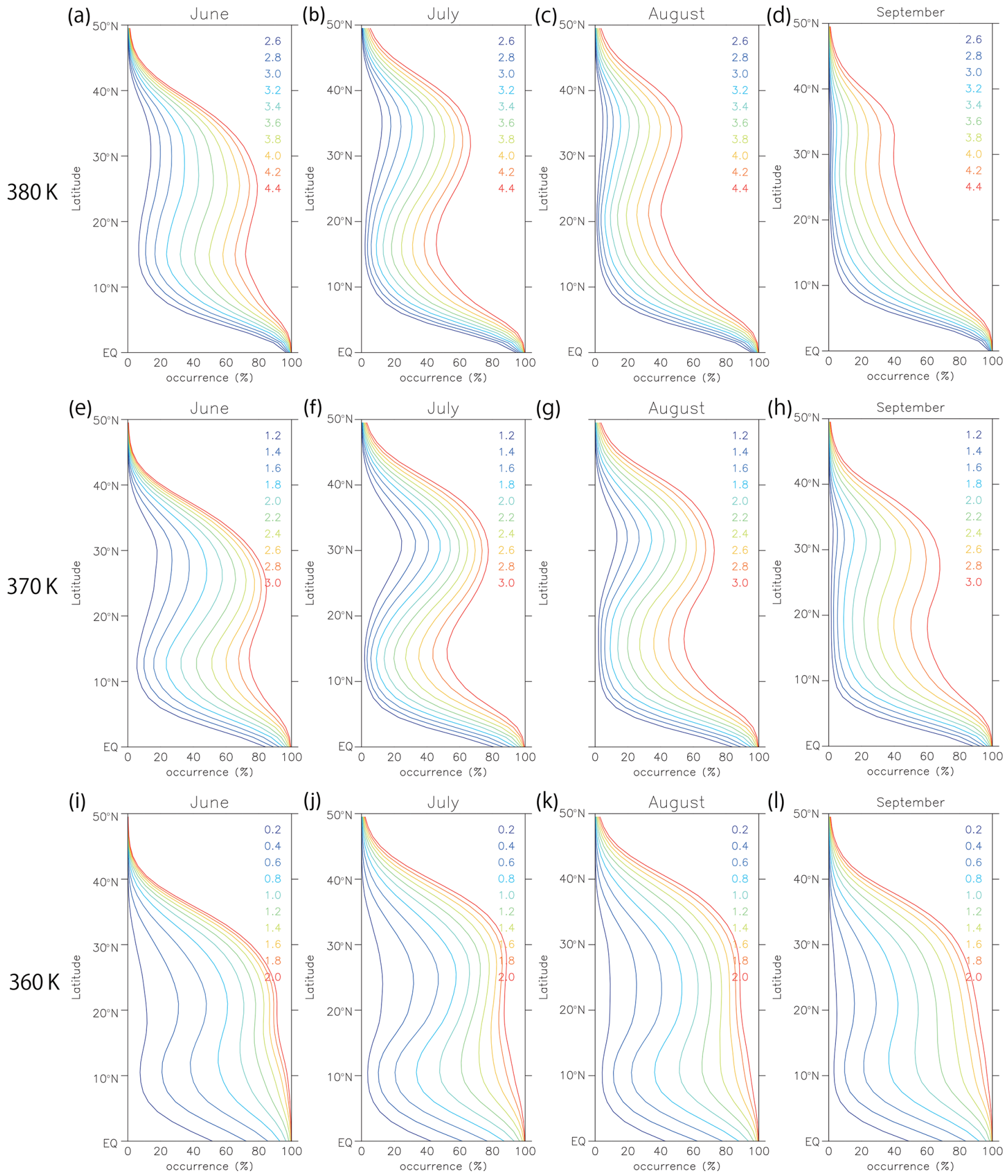

Figure A1. Percentage of the occurrence of grid points where PV is below the reference value between 0 and $160^{\circ} \mathrm{E}$, as functions of latitude, calculated using ERA-Interim data from 1979 to 2016. Different line colors correspond to the different reference PV values that are shown in the top right of each figure. 


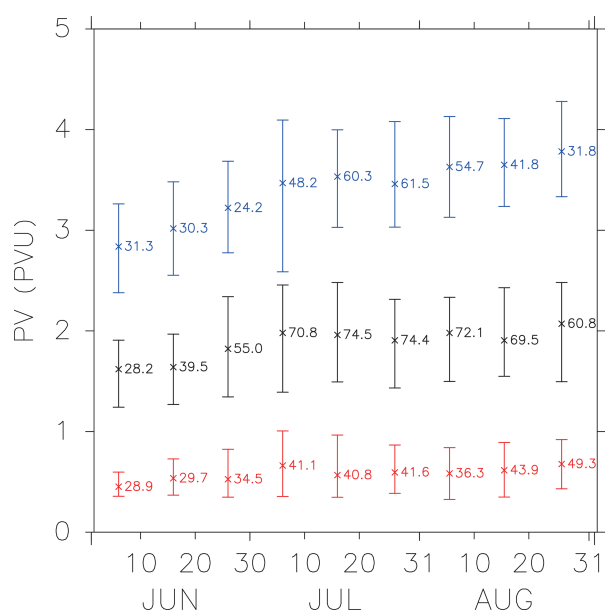

Figure A2. Statistics for the daily barrier PV values, determined objectively by the method of Ploeger et al. (2015), using ERA-Interim reanalysis data from 1979 to 2016 . The horizontal axis is the time of year, with nine time ranges corresponding to the early, middle, and late periods of each month between June and August. Red, black, and blue lines respectively show the results for the 360, 370, and $380 \mathrm{~K}$ levels. Vertical lines show the range of PV values between 15 th and 85th percentiles, with crosses in the middle corresponding to the median values. The numbers next to the crosses represent corresponding percentages of the cases when the barrier is successfully determined.

ues implied by Fig. A1 to detect anticyclonic air sufficiently widely and also separated from the equatorial air.

Supported by these results, we chose the calculation domain and reference values in the following. The domain is chosen to be inside $12-50^{\circ} \mathrm{N}, 10^{\circ} \mathrm{W}-160^{\circ} \mathrm{E}$. The reference $\mathrm{PV}$ value used for analyses was chosen to be $2.0 \mathrm{PVU}$ on $370 \mathrm{~K}$. The high percentage of barrier detection around this value implies the relevance of the underlying concept of an isolated strong nearly inviscid anticyclone, in which low-PV air is surrounded by a distinct boundary with a steep PV gradient. For the purpose of the comparison of the zonal anticyclone area flux on different levels, reference values 0.5 and 3.5 PVU were also used respectively on 360 and $380 \mathrm{~K}$. Most analyses were performed for July and August, when the anticyclone is the most intense and the PV-based definition is the most relevant. Three-month data from June to August are only used for the spectrum analyses.

\section{Appendix B: Derivation of the tendency equation for the thickness-weighted low-PV area}

Equation (3) can be derived in a way similar to the derivation of Eq. (13) of Butchart and Remsberg (1986). For consistency, their notation is used in the following. They begin with the small change in the area $\Delta A(t)_{\chi \geq \chi_{0}}$ enclosed by an isopleth $\chi=\chi_{0}$, denoted by $\Gamma$, with respect to the change in the contour position $\Delta \boldsymbol{x}$. Note that they assumed that the value of $\chi$ increases inward of the area.

$$
-\oint_{\Gamma} \Delta \boldsymbol{x} \cdot \frac{\nabla_{\theta} \chi}{\left|\nabla_{\theta} \chi\right|} \mathrm{d} s=\Delta A(t)_{\chi \geq \chi_{0}}
$$

To extend this formulation to the thickness-weighted area $\hat{A}(t)_{\chi \geq \chi_{0}}$, there needs an additional term for thickness change $\Delta \sigma$ in the left-hand side,

$$
\int_{\chi \geq \chi_{0}} \Delta \sigma \mathrm{d} A-\oint_{\Gamma} \Delta \boldsymbol{x} \cdot \frac{\nabla_{\theta} \chi}{\left|\nabla_{\theta} \chi\right|} \sigma \mathrm{d} s=\Delta \hat{A}(t)_{\chi \geq \chi_{0}} .
$$

Using the equation for thickness

$$
\frac{\partial \sigma}{\partial t}+\nabla_{\theta} \cdot(\sigma \boldsymbol{v})=-\frac{\partial}{\partial \theta}(\sigma \dot{\theta})
$$

the first term on the left-hand side of Eq. (B2) can be rewritten as follows:

$$
\begin{aligned}
\int_{\chi \geq \chi_{0}} \Delta \sigma \mathrm{d} A & =\Delta t\left[-\int_{\chi \geq \chi_{0}} \nabla_{\theta} \cdot(\sigma \boldsymbol{v}) \mathrm{d} A-\int_{\chi \geq \chi_{0}} \frac{\partial}{\partial \theta}(\sigma \dot{\theta}) \mathrm{d} A\right] \\
& =\Delta t\left[\oint_{\Gamma} \boldsymbol{v} \cdot \frac{\nabla_{\theta} \chi}{\left|\nabla_{\theta} \chi\right|} \sigma \mathrm{d} s-\int_{\chi \geq \chi_{0}} \frac{\partial}{\partial \theta}(\sigma \dot{\theta}) \mathrm{d} A\right],
\end{aligned}
$$

where $v$ is two-dimensional velocity, and $\nabla_{\theta}$ is the twodimensional gradient on an isentropic surface.

The second term on the right-hand side is transformed using $\Delta \boldsymbol{x} \cdot \nabla_{\theta} \chi-\Delta t \cdot \partial \chi / \partial t$ as shown in Butchart and Remsberg (1986). Then by applying $\Delta t \rightarrow \mathrm{d} t$ we obtain the following:

$$
\begin{aligned}
\frac{\mathrm{d}}{\mathrm{d} t} \hat{A}(t)_{\chi \geq \chi_{0}} & =\oint_{\Gamma}\left(\frac{\partial \chi}{\partial t}+\boldsymbol{v} \cdot \nabla_{\theta} \chi\right) \frac{\sigma \mathrm{d} s}{\left|\nabla_{\theta} \chi\right|} \\
& -\int_{\chi \geq \chi_{0}} \frac{\partial}{\partial \theta}(\sigma \dot{\theta}) \mathrm{d} A .
\end{aligned}
$$

As the first integral contains the advection term, the bracket can be replaced with a nonconservation term $F$.

$$
\frac{\partial \chi}{\partial t}+\boldsymbol{v} \cdot \nabla_{\theta} \chi=F
$$

When $\chi$ is potential vorticity and the area $A$ is defined to have potential vorticity below the reference value, Eq. (3) is obtained. The subgrid-scale mixing term in Butchart and Remsberg (1986) comes from the difference between the true divergence term and the divergence term calculated from resolved variables. Although our equation does not have a divergence term, we consider it is still better to include the unresolved effect such as subgrid-scale mixing, which is included in $F$. Then, when $\chi$ is potential vorticity and the area $A$ is defined to have potential vorticity below the reference value, using

$F=-q \frac{\partial \dot{\theta}}{\partial \theta}+\dot{\theta} \frac{\partial q}{\partial \theta}+($ unresolved term $)$ 


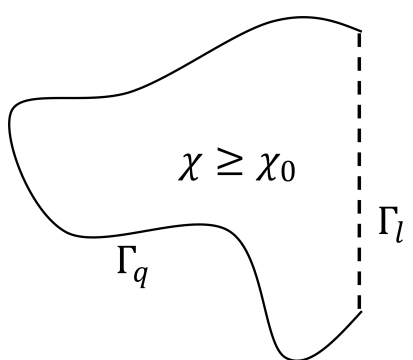

Figure B1. An example of the area enclosed by the isopleth of $\chi$ and a specific longitude. $\Gamma_{q}$ is the part of the isopleth $\chi=\chi_{0} . \Gamma_{l}$ is the part of the circle of longitude $\lambda=\lambda_{0}$.

we thus obtain Eq. (3).

Equation (3) can also be derived from the general mass conservation expression in a PV- $\theta$ coordinate introduced in Nakamura (1995):

$$
\left(\frac{\partial m}{\partial t}\right)_{q, \theta}+\left(\frac{\partial \mathcal{M}(\dot{q})}{\partial q}\right)_{\theta, t}+\left(\frac{\partial \mathcal{M}(\dot{\theta})}{\partial \theta}\right)_{q, t}=0,
$$

where $m=\mathcal{M}(1)$ and the thickness-weighted area integration operator $\mathcal{M}$ is defined as

$\mathcal{M}(*)=\iint_{q \leq q_{0}}(*) \sigma \mathrm{d} A=\int_{q^{*} \leq q} \mathrm{~d} q^{*} \oint_{q *}(*) \frac{\sigma \mathrm{d} s}{\left|\nabla_{\theta} q^{*}\right|}$.

Substituting $\dot{q}=F$ and expanding $\mathcal{M}(\dot{q})$ and $\mathcal{M}(\dot{\theta})$, we obtain Eq. (3).

Next, let us derive Eq. (5). Now suppose the small change of the area $\Delta \hat{A}(t)_{\chi \geq \chi_{0}, \lambda \leq \lambda_{0}}$ enclosed by the isopleth $\chi=\chi_{0}$ and the circle of longitude $\lambda_{0}$. Let $\Gamma_{q}$ and $\Gamma_{l}$ respectively be the isopleth and the circle of longitude consisting the border of the area as shown in Fig. (B1). Equation (B1) is modified as follows:

$$
\int_{\chi \geq \chi_{0}} \Delta \sigma \mathrm{d} A-\oint_{\Gamma_{q}} \Delta \boldsymbol{x} \cdot \frac{\nabla_{\theta \chi}}{\left|\nabla_{\theta \chi}\right|} \sigma \mathrm{d} s=\Delta \hat{A}(t)_{\chi \geq \chi_{0}, \lambda \leq \lambda_{0}} .
$$

The integral of the first term on the left-hand side is performed over the area $\Delta \hat{A}(t)_{\chi \geq \chi_{0}, \lambda \leq \lambda_{0}}$, and the line integral of the second term is performed only over $\Gamma_{q}$, as $\Gamma_{l}$ is constant with time. The first term can be rewritten as follows, using Gauss' theorem for $\Gamma_{q}$ and $\Gamma_{l}$ :

$$
\begin{aligned}
\int_{\chi \geq \chi_{0}, \lambda \leq \lambda_{0}} \Delta \sigma \mathrm{d} A & =\Delta t\left[-\int_{\chi \geq \chi_{0}} \nabla_{\theta} \cdot(\sigma \boldsymbol{v}) \mathrm{d} A\right. \\
& \left.-\int_{\chi \geq \chi_{0}} \frac{\partial}{\partial \theta}(\sigma \dot{\theta}) \mathrm{d} A\right], \\
& =\Delta t\left[\int_{\Gamma_{q}} \boldsymbol{v} \cdot \frac{\nabla_{\theta} \chi}{\left|\nabla_{\theta} \chi\right|} \sigma \mathrm{d} s+\int_{\Gamma_{l}} u \sigma \mathrm{d} s\right. \\
& \left.-\int_{\chi \geq \chi_{0}} \frac{\partial}{\partial \theta}(\sigma \dot{\theta}) \mathrm{d} A\right] .
\end{aligned}
$$

Then we obtain Eq. (5) with the additional term $\hat{F}(\lambda)$ defined as follows. The integral is performed over the longitude circle $\lambda_{0}$ consisting the border of the area.

$$
\hat{F}(\lambda)=\int_{q \leq q_{0}, \lambda_{0}} u \sigma \mathrm{d} s=\int_{q \leq q_{0}, \lambda_{0}} u \sigma R \mathrm{~d} \phi
$$


Data availability. The ERA-Interim data were downloaded from the ECMWF data server (http://apps.ecmwf.int/datasets/, Dee et al., 2011). Daily outgoing longwave radiation (OLR) data were downloaded from the NOAA PSL server (https://psl.noaa.gov/data/ gridded/data.interp_OLR.html, Liebmann and Smith, 1996).

Author contributions. AA conducted the data analysis. AA and KS contributed to the discussion and the writing of the paper.

Competing interests. The authors declare that they have no conflict of interest.

Acknowledgements. The authors thank two anonymous reviewers for their constructive comments. This study is supported by the Japan Science and Technology Agency CREST program (JPMJCR 1663 ) and the Japan Society for the Promotion of Science (JSPS) Grant-in-Aid Scientific Research (A) 25247075 program. All of the figures were prepared using fortran DCL, developed by GFD Dennou Club (https://www.gfd-dennou.org/index.html.en, last access: 26 April 2020).

Financial support. This research has been supported by the Japan Science and Technology Agency, Core Research for Evolutional Science and Technology (grant no. JPMJCR 1663), and the Japan Society for the Promotion of Science (grant no. Grant-in-Aid Scientific Research (A) 25247075).

Review statement. This paper was edited by Marc von Hobe and reviewed by Bernard Legras and one anonymous referee.

\section{References}

Amemiya, A. and Sato, K.: A two-dimensional dynamical model for the subseasonal variability of the Asian monsoon anticyclone, J. Atmos. Sci., 75, 3597-3612, 2018.

Annamalai, H. and Slingo, J.: Active/break cycles: diagnosis of the intraseasonal variability of the Asian summer monsoon, Clim. Dynam., 18, 85-102, 2001.

Bian, J., Pan, L. L., Paulik, L., Vømel, H., Chen, H., and Lu, D.: In situ water vapor and ozone measurements in Lhasa and Kunming during the Asian summer monsoon, Geophys. Res. Lett., 39, L19808, https://doi.org/10.1029/2012GL052996, 2012.

Branstator, G. and Teng, H.: Tropospheric Waveguide Teleconnections and Their Seasonality, J. Atmos. Sci., 74, 1513-1532, 2017.

Butchart, N. and Remsberg, E. E.: The area of the stratospheric polar vortex as a diagnostic for tracer transport on an isentropic surface, J. Atmos. Sci., 43, 1319-1339, 1986.

Chen, B., Xu, X. D., Yang, S., and Zhao, T. L.: Climatological perspectives of air transport from atmospheric boundary layer to tropopause layer over Asian monsoon regions during boreal summer inferred from Lagrangian approach, Atmos.
Chem. Phys., 12, 5827-5839, https://doi.org/10.5194/acp-125827-2012, 2012.

Dee, D. P., Uppala, S. M., Simmons, A. J., Berrisford, P., Poli, P., Kobayashi, S., Andrae, U., Balmaseda, M. A., Balsamo, G., Bauer, P., Bechtold, P., Beljaars, A. C. M., van de Berg, L., Bidlot, J., Bormann, N., Delsol, C., Dragani, R., Fuentes, M., Geer, A. J., Haimberger, L., Healy, S. B., Hersbach, H., Hólm, E. V., Isaksen, L., Kållberg, P., Köhler, M., Matricardi, M., McNally, A. P., Monge-Sanz, B. M., Morcrette, J.-J., Park, B.-K., Peubey, C., de Rosnay, P., Tavolato, C., Thépaut, J.-N., and Vitart, F.: The ERA-Interim reanalysis: Configuration and performance of the data assimilation system, Q. J. Roy. Meteor. Soc., 137, 553597, https://doi.org/10.1002/qj.828, 2011 (data available at: http: //apps.ecmwf.int/datasets/, last access: 26 April 2020).

Dethof, A., O’Neill, A., Slingo, J., and Smit, H.: A mechanism for moistening the lower stratosphere involving the Asian summer monsoon, Q. J. Roy. Meteor. Soc., 125, 1079-1106, 1999.

Ding, Q. and Wang, B.: Circumglobal teleconnection in the Northern Hemisphere summer, J. Climate, 18, 3483-3505, 2005.

Ding, Q. and Wang, B.: Intraseasonal teleconnection between the summer Eurasian wave train and the Indian monsoon, J. Climate, 20, 3751-3767, 2007.

Ding, Q. and Wang, B.: Predicting extreme phases of the Indian summer monsoon, J. Climate, 22, 346-363, 2009.

Dunkerton, T. J.: Evidence of meridional motion in the summer lower stratosphere adjacent to monsoon regions, J. Geophys. Res.-Atmos., 100, 16675-16688, 1995.

Fadnavis, S., Roy, C., Chattopadhyay, R., Sioris, C. E., Rap, A., Müller, R., Kumar, K. R., and Krishnan, R.: Transport of trace gases via eddy shedding from the Asian summer monsoon anticyclone and associated impacts on ozone heating rates, Atmos. Chem. Phys., 18, 11493-11506, https://doi.org/10.5194/acp-1811493-2018, 2018.

Fujinami, H. and Yasunari, T.: Submonthly variability of convection and circulation over and around the Tibetan Plateau during the boreal summer, J. Meteorol. Soc. Jpn. Ser. II, 82, 1545-1564, 2004.

Garny, H. and Randel, W.: Dynamic variability of the Asian monsoon anticyclone observed in potential vorticity and correlations with tracer distributions, J. Geophys. Res.-Atmos., 118, 13-421, 2013.

Garny, H. and Randel, W. J.: Transport pathways from the Asian monsoon anticyclone to the stratosphere, Atmos. Chem. Phys., 16, 2703-2718, https://doi.org/10.5194/acp-16-27032016, 2016.

Gettelman, A., Kinnison, D. E., Dunkerton, T. J., and Brasseur, G. P.: Impact of monsoon circulations on the upper troposphere and lower stratosphere, J. Geophys. Res.-Atmos., 109, D22101, https://doi.org/10.1029/2004JD004878, 2004.

Gottschaldt, K.-D., Schlager, H., Baumann, R., Cai, D. S., Eyring, V., Graf, P., Grewe, V., Jöckel, P., Jurkat-Witschas, T., Voigt, C., Zahn, A., and Ziereis, H.: Dynamics and composition of the Asian summer monsoon anticyclone, Atmos. Chem. Phys., 18, 5655-5675, https://doi.org/10.5194/acp-18-5655-2018, 2018.

Hoskins, B. J., McIntyre, M., and Robertson, A. W.: On the use and significance of isentropic potential vorticity maps, Q. J. Roy. Meteor. Soc., 111, 877-946, 1985. 
Hsu, C. J. and Plumb, R. A.: Nonaxisymmetric thermally driven circulations and upper-tropospheric monsoon dynamics, J. Atmos. Sci., 57, 1255-1276, 2000.

Kosaka, Y., Nakamura, H., Watanabe, M., and Kimoto, M.: Analysis on the dynamics of a wave-like teleconnection pattern along the summertime Asian jet based on a reanalysis dataset and climate model simulations, J. Meteorol. Soc. Jpn. Ser. II, 87, 561580, 2009.

Krishnamurti, T., Daggupaty, S., Fein, J., Kanamitsu, M., and Lee, J. D.: Tibetan high and upper tropospheric tropical circulations during northern summer, B. Am. Meteorol. Soc., 54, 1234-1250, 1973.

Lee, J.-Y., Wang, B., Wheeler, M. C., Fu, X., Waliser, D. E., and Kang, I.-S.: Real-time multivariate indices for the boreal summer intraseasonal oscillation over the Asian summer monsoon region, Clim. Dynam., 40, 493-509, 2013.

Liebmann, B. and Smith, C. A.: Description of a complete (interpolated) outgoing longwave radiation dataset, B. Am. Meteorol. Soc., 77, 1275-1277, 1996 (data available at: https: //psl.noaa.gov/data/gridded/data.interp_OLR.html, last access: 26 April 2020).

Liu, Y., Hoskins, B., and Blackburn, M.: Impact of Tibetan orography and heating on the summer flow over Asia, J. Meteorol. Soc. Jpn. Ser. II, 85, 1-19, 2007.

Luo, J., Pan, L. L., Honomichl, S. B., Bergman, J. W., Randel, W. J., Francis, G., Clerbaux, C., George, M., Liu, X., and Tian, W.: Space-time variability in UTLS chemical distribution in the Asian summer monsoon viewed by limb and nadir satellite sensors, Atmos. Chem. Phys., 18, 12511-12530, https://doi.org/10.5194/acp-18-12511-2018, 2018.

Nakamura, N.: Modified Lagrangian-mean diagnostics of the stratospheric polar vortices. Part I. Formulation and analysis of GFDL SKYHI GCM, J. Atmos. Sci., 52, 2096-2108, 1995.

Nakamura, N.: Two-dimensional mixing, edge formation, and permeability diagnosed in an area coordinate, J. Atmos. Sci., 53, 1524-1537, 1996.

Nash, E. R., Newman, P. A., Rosenfield, J. E., and Schoeberl, M. R.: An objective determination of the polar vortex using Ertel's potential vorticity, J. Geophys. Res.-Atmos., 101, 9471-9478, 1996.

North, G. R., Bell, T. L., Cahalan, R. F., and Moeng, F. J.: Sampling errors in the estimation of empirical orthogonal functions, Mon. Weather Rev., 110, 699-706, 1982.

Norton, W. A.: Breaking Rossby waves in a model stratosphere diagnosed by a vortex-following coordinate system and a technique for advecting material contours, J. Atmos. Sci., 51, 654673,1994

Nützel, M., Dameris, M., and Garny, H.: Movement, drivers and bimodality of the South Asian High, Atmos. Chem. Phys., 16, 14755-14774, https://doi.org/10.5194/acp-16-14755-2016, 2016.

Ortega, S., Webster, P. J., Toma, V., and Chang, H.-R.: Quasibiweekly oscillations of the South Asian monsoon and its coevolution in the upper and lower troposphere, Clim. Dynam., 49, 1-16, https://doi.org/10.1007/s00382-016-3503-y, 2017.

Ortega, S., Webster, P. J., Toma, V., and Chang, H. R.: The effect of potential vorticity fluxes on the circulation of the tropical upper troposphere, Q. J. Roy. Meteor. Soc., 144, 848-860, https://doi.org/10.1002/qj.3261, 2018.
Pan, L. L., Kunz, A., Homeyer, C. R., Munchak, L. A., Kinnison, D. E., and Tilmes, S.: Commentary on using equivalent latitude in the upper troposphere and lower stratosphere, Atmos. Chem. Phys., 12, 9187-9199, https://doi.org/10.5194/acp12-9187-2012, 2012.

Pan, L. L., Honomichl, S. B., Kinnison, D. E., Abalos, M., Randel, W. J., Bergman, J. W., and Bian, J.: Transport of chemical tracers from the boundary layer to stratosphere associated with the dynamics of the Asian summer monsoon, J. Geophys. Res.-Atmos., 121, 14159-14174, https://doi.org/10.1002/2016JD025616, 2016.

Park, M., Randel, W. J., Gettelman, A., Massie, S. T., and Jiang, J. H.: Transport above the Asian summer monsoon anticyclone inferred from Aura Microwave Limb Sounder tracers, J. Geophys. Res.-Atmos., 112, D16309, https://doi.org/10.1029/2006JD008294, 2007.

Park, M., Randel, W. J., Emmons, L. K., Bernath, P. F., Walker, K. A., and Boone, C. D.: Chemical isolation in the Asian monsoon anticyclone observed in Atmospheric Chemistry Experiment (ACE-FTS) data, Atmos. Chem. Phys., 8, 757-764, https://doi.org/10.5194/acp-8-757-2008, 2008.

Park, M., Randel, W. J., Emmons, L. K., and Livesey, N. J.: Transport pathways of carbon monoxide in the Asian summer monsoon diagnosed from Model of Ozone and Related Tracers (MOZART), J. Geophys. Res.-Atmos., 114, D08303, https://doi.org/10.1029/2008JD010621, 2009.

Ploeger, F., Gottschling, C., Griessbach, S., Grooß, J.-U., Guenther, G., Konopka, P., Müller, R., Riese, M., Stroh, F., Tao, M., Ungermann, J., Vogel, B., and von Hobe, M.: A potential vorticitybased determination of the transport barrier in the Asian summer monsoon anticyclone, Atmos. Chem. Phys., 15, 13145-13159, https://doi.org/10.5194/acp-15-13145-2015, 2015.

Popovic, J. M. and Plumb, R. A.: Eddy shedding from the uppertropospheric Asian monsoon anticyclone, J. Atmos. Sci., 58, 93 104, 2001.

Randel, W. J. and Park, M.: Deep convective influence on the Asian summer monsoon anticyclone and associated tracer variability observed with Atmospheric Infrared Sounder (AIRS), J. Geophys. Res.-Atmos., 111, D12314, https://doi.org/10.1029/2005JD006490, 2006.

Randel, W. J., Park, M., Emmons, L., Kinnison, D., Bernath, P., Walker, K. A., Boone, C., and Pumphrey, H.: Asian monsoon transport of pollution to the stratosphere, Science, 328, 611-613, 2010.

Santee, M., Manney, G., Livesey, N., Schwartz, M., Neu, J., and Read, W.: A comprehensive overview of the climatological composition of the Asian summer monsoon anticyclone based on 10 years of Aura Microwave Limb Sounder measurements, J. Geophys. Res.-Atmos., 122, 5491-5514, 2017.

Terao, T.: Barotropic disturbances on intraseasonal time scales observed in the midlatitudes over the Eurasian continent during the northern summer, J. Meteorol. Soc. Jpn. Ser. II, 76, 419-436, 1998.

Vogel, B., Günther, G., Müller, R., Grooß, J.-U., Hoor, P., Krämer, M., Müller, S., Zahn, A., and Riese, M.: Fast transport from Southeast Asia boundary layer sources to northern Europe: rapid uplift in typhoons and eastward eddy shedding of the Asian monsoon anticyclone, Atmos. Chem. Phys., 14, 12745-12762, https://doi.org/10.5194/acp-14-12745-2014, 2014. 
Vogel, B., Günther, G., Müller, R., Grooß, J.-U., Afchine, A., Bozem, H., Hoor, P., Krämer, M., Müller, S., Riese, M., Rolf, C., Spelten, N., Stiller, G. P., Ungermann, J., and Zahn, A.: Longrange transport pathways of tropospheric source gases originating in Asia into the northern lower stratosphere during the Asian monsoon season 2012, Atmos. Chem. Phys., 16, 15301-15325, https://doi.org/10.5194/acp-16-15301-2016, 2016.

Vogel, B., Müller, R., Günther, G., Spang, R., Hanumanthu, S., Li, D., Riese, M., and Stiller, G. P.: Lagrangian simulations of the transport of young air masses to the top of the Asian monsoon anticyclone and into the tropical pipe, Atmos. Chem. Phys., 19, 6007-6034, https://doi.org/10.5194/acp-19-6007-2019, 2019.
Wei, W., Zhang, R., Yang, S., Li, W., and Wen, M.: Quasi-Biweekly Oscillation of the South Asian High and Its Role in Connecting the Indian and East Asian Summer Rainfalls, Geophys. Res. Lett., 46, 14742-14750, https://doi.org/10.1029/2019GL086180, 2019.

Zhang, Q., Guoxiong, W., and Yongfu, Q.: The bimodality of the $100 \mathrm{hPa}$ South Asia High and its relationship to the climate anomaly over East Asia in summer, J. Meteorol. Soc. Jpn. Ser. II, 80, 733-744, 2002. 